\title{
Holographic quantum circuits from splitting/joining local quenches
}

\author{
Teppei Shimaji, ${ }^{a}$ Tadashi Takayanagi $^{a, b}$ and Zixia Wei ${ }^{a}$ \\ ${ }^{a}$ Center for Gravitational Physics, Yukawa Institute for Theoretical Physics, \\ Kyoto University, Kyoto 606-8502, Japan \\ ${ }^{b}$ Kavli Institute for the Physics and Mathematics of the Universe (WPI), \\ University of Tokyo, Kashiwa, Chiba 277-8582, Japan \\ E-mail: teppei.shimaji@yukawa.kyoto-u.ac.jp, \\ takayana@yukawa.kyoto-u.ac.jp, zixia.wei@yukawa.kyoto-u.ac.jp
}

ABstract: We study three different types of local quenches (local operator, splitting and joining) in both the free fermion and holographic CFTs in two dimensions. We show that the computation of a quantity called entanglement density, provides a systematic method to capture essential properties of local quenches. This allows us to clearly understand the differences between the free and holographic CFTs as well as the distinctions between three local quenches. We also analyze holographic geometries of splitting/joining local quenches using the AdS/BCFT prescription. We show that they are essentially described by time evolutions of boundary surfaces in the bulk AdS. We find that the logarithmic time evolution of entanglement entropy arises from the region behind the Poincaré horizon as well as the evolutions of boundary surfaces. In the CFT side, our analysis of entanglement density suggests such a logarithmic growth is due to initial non-local quantum entanglement just after the quench. Finally, by combining our results, we propose a new class of gravity duals, which are analogous to quantum circuits or tensor networks such as MERA, based on the AdS/BCFT construction.

Keywords: AdS-CFT Correspondence, Conformal Field Theory, Holography and condensed matter physics (AdS/CMT)

ARXIV EPRINT: 1812.01176 


\section{Contents}

1 Introduction $\quad 2$

2 Entanglement entropy and AdS/(B)CFT 3

2.1 EE for CFT vacuum in flat space 3

2.2 EE for excited states from conformal transformation 5

2.3 Conformal map in $\mathrm{AdS}_{3} / \mathrm{CFT}_{2} \quad 5$

2.4 Holographic entanglement entropy in $\mathrm{AdS}_{3} / \mathrm{CFT}_{2} \quad 6$

2.5 EE from AdS/BCFT 6

3 Entanglement density $\quad 8$

3.1 Properties 9

$\begin{array}{llr}3.2 & \text { Example 1: global quenches } & 10\end{array}$

3.3 Example 2: local operator quenches 11

4 Splitting local quench $\quad \mathbf{1 4}$

$\begin{array}{lll}4.1 & \text { Splitting local quench in Dirac free fermion CFT } & 15\end{array}$

$\begin{array}{lll}4.2 & \text { Splitting local quench in holographic CFT } & 17\end{array}$

4.3 Holographic geometry of splitting local quench 20

4.4 Splitting local quench in a simple spin system 24

5 Joining local quench $\quad \mathbf{2 5}$

5.1 Joining local quench in Dirac free fermion CFT 26

$\begin{array}{lll}5.2 & \text { Joining local quench in holographic CFT }\end{array}$

$\begin{array}{lll}5.3 & \text { Holographic geometry of joining local quench } & 30\end{array}$

$6 \quad \log t$ geodesic length in holographic Lorentzian geometry 32

$\begin{array}{lll}6.1 & \text { Late time behavior } & 32\end{array}$

6.2 Middle time period 36

$\begin{array}{lll}7 & \text { Tensor network interpretation of local quenches } & \mathbf{3 6}\end{array}$

$\begin{array}{lll}7.1 & \text { Local operator quench } & 38\end{array}$

$\begin{array}{ll}7.2 & \text { Splitting quench } \\ 7.3 & \text { Joining quench }\end{array}$

$\begin{array}{lll}7.3 & \text { Joining quench } & 40\end{array}$

8 Holographic quantum circuits from AdS/BCFT 41

9 Conclusions $\quad 42$

A Vacuum entanglement density in massless Dirac fermion CFT 44

B Detailed computations of evolutions of holographic entanglement entropy 


\section{Introduction}

Conformal field theories (CFTs) in two dimensions are very special among quantum field theories (QFTs) in that infinite dimensional conformal symmetries constrain their properties. Owing to this, we can analytically calculate much more physical quantities than those in ordinary QFTs. Nevertheless, we can observe a variety of qualitative difference in the dynamics of two dimensional CFTs (2d CFTs). We can easily come up with two extreme examples of 2d CFTs. One is free CFTs such as massless free fermion or scalar theories. Another one is the strong coupling limits of $2 \mathrm{~d}$ CFTs with large central charges $c$, namely the holographic CFTs, which have dual descriptions via the AdS/CFT correspondence [1-3].

One of the most interesting quantities to characterize the dynamical property of a given quantum state is the entanglement entropy (EE) [4-10]. In this paper we would like to explore how differences of $2 \mathrm{~d}$ CFTs appear in the time evolution of certain excited states. One useful class of excited states is called local quenches, where we exert a local excitation on a vacuum state. Since it initially modifies the state only locally, they provide clean examples where we can interpret the time evolution easier. We consider three different types of local quenches: (i) local operator quenches, (ii) splitting local quenches, and (iii) joining local quenches. We sketched them in figure 1.

The first one (i) is simply defined by acting a local operator on a vacuum and we analyze its time evolution. This was first introduced in $[11,12]$ and there have already been many related results both in field theory analysis and holographic analysis. The third one (iii) is defined by joining two semi-infinite lines, as first introduced in [13]. The second one (ii) is triggered by splitting a connected line into two disconnected ones. This is a new setup which the present paper will discuss in detail.

In this paper we will point out that it is very helpful and systematic to analyze not the entanglement entropy itself but its second derivatives, called the entanglement density (ED) [14]. Indeed, this quantity extracts the essential behaviors of entanglement entropy under local quenches in term of clear peaks in its graph. By studying the behavior of entanglement density, we can clearly see both similarities and differences among the above three types of local quenches in free CFTs and holographic CFTs, as we will explain later. In particular, this observation resolves an apparent puzzle on the known logarithmic growing entanglement entropy under holographic operator local quenches.

Another purpose of this paper is to explore the spacetime geometries of holographic local quenches. Since the gravity dual geometry of (i) local operator quenches was already given in [14], we will focus on (ii) and (iii). For (iii) joining local quenches, the construction of gravity dual by employing the AdS/BCFT formulation $[15,16]$ was given in [17] and we will study more details of the spacetime geometry by using this description in this paper. In addition we will provide the gravity dual geometry for (ii) splitting local quenches. Refer to [18] for other classes of topology changing quantum operations in CFTs such as projections and partial identifications. Our details geometric analysis clearly will explain the two different sources of logarithmic time evolutions of entanglement entropy observed in the local quenches. 
Our analysis of holographic geometry of local quenches is also motivated by the conjectured connection between the AdS/CFT and tensor networks [19] such as the MERA [20, 21]. In tensor network descriptions, we normally consider discretized lattice theories whose continuum limits correspond to CFTs. Therefore, it is not directly related to the continuous AdS spacetimes, but to their discretized versions as in $[19,22,23]$. One way to resolve this problem is to consider a continuous tensor networks as in the continuous MERA [24-26] or path-integral approaches [27-34].

However, it is also intriguing to try to realize a discretized version of AdS in a way that it is naturally derived from the conventional AdS/CFT. The gravity duals of local quenches are useful for this purpose because we can model a class of quantum gates in tensor networks by arranging the splitting and joining procedures in CFTs. As we will discuss in the final part of this paper, we presents a sketch of gravity duals of MERA tensor networks, by combining the holographic local quenches. This indeed qualitatively supports the conjecture.

This paper is organized as follows: in section 2, we review basic methods of calculating entanglement entropy by conformal map and also by AdS/(B)CFT. In section 3, after we review the definition of entanglement density, we study its behaviors for global and local operator quenches. In section 4 , we analyze the splitting local quenches and its holographic dual. In section 5, we analyze the joining local quenches and its holographic dual. In section 6 , we explain how the logarithmic growth of entanglement entropy arises from a geodesic length in each gravity dual. In section 7 , we provide qualitative tensor network description of local quenches. In section 8, we combine the results in this paper to provide a new gravity dual of a MERA-like (discretized) tensor network, in the framework of AdS/BCFT. In section 9, we summarize our conclusions and discuss future problems. In appendix A, we show the entanglement density can reproduce the correct entanglement entropy even when the subsystem consists of multiple disconnected intervals in two dimensional massless Dirac fermion CFT. In appendix B, we will present the detailed computations of time evolutions of holographic entanglement entropy under splitting/joining local quenches.

\section{Entanglement entropy and AdS/(B)CFT}

Here we briefly review calculations of entanglement entropy (EE) in two dimensional CFTs, based on conformal mappings and computations of holographic entanglement entropy (HEE) based on the AdS/CFT and AdS/BCFT. The entanglement entropy is defined by the von-Neumann entropy $S_{A}=-\operatorname{Tr}\left[\rho_{A} \log \rho_{A}\right]$, where $\rho_{A}$ is the reduced density matrix defined by tracing out the original quantum state over all parts of Hilbert space other than $A$.

\subsection{EE for CFT vacuum in flat space}

Consider a two dimensional CFT (2d CFT) on a plane $R^{2}$, which is described by the complex coordinate $(w, \bar{w})$. We define the time and space coordinate $(\tau, x)$ as

$$
w=x+i \tau, \quad \bar{w}=x-i \tau .
$$




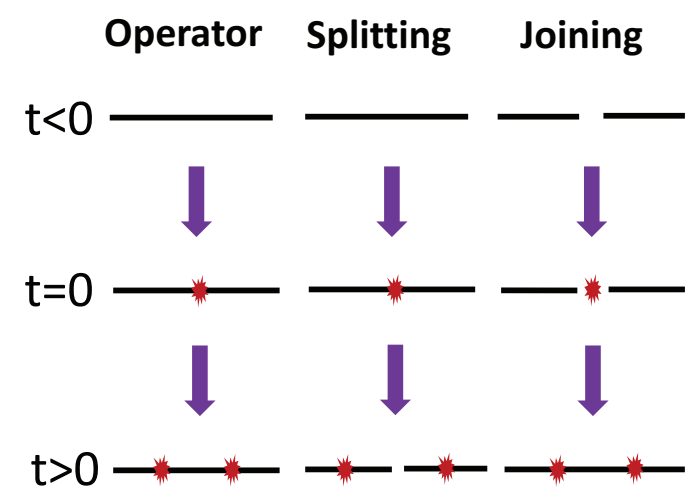

Figure 1. The three local quenches are sketched: the local operator quench (left), the splitting local quench (middle), and the joining local quench (right) in two dimensional CFTs. The red points are locations where the energy density is very large.

The time $\tau$ is Euclidean time and is analytically continued to the real time by

$$
\tau=i t
$$

A two point function of a primary operator $O$ in a $2 \mathrm{~d}$ CFT behaves as

$$
\left\langle O\left(w_{1}, \bar{w}_{1}\right) O\left(w_{2}, \bar{w}_{2}\right)\right\rangle=\frac{1}{\left|w_{1}-w_{2}\right|^{2(h+\bar{h})}},
$$

where the primary operator $O$ has the chiral/anti-chiral conformal dimension given by $(h, \bar{h})$. For the twist operator $\sigma_{n}$, we have $h=\bar{h}=\frac{c}{24}(n-1 / n)$. When we compute the entanglement entropy (EE) for the subsystem $A$ defined by the interval $0 \leq x \leq l$ at a fixed time $\tau=0$, the relevant two point function is

$$
\left\langle\sigma_{n}(l, l) \bar{\sigma}_{n}(0,0)\right\rangle=\frac{1}{l^{\frac{c}{6}(n-1 / n)}} .
$$

Therefore the EE $S_{A}$ for the CFT vacuum reads

$$
S_{A}=-\left.\frac{\partial}{\partial n} \log \left\langle\sigma_{n}(l, l) \bar{\sigma}_{n}(0,0)\right\rangle\right|_{n=1}=\frac{c}{3} \log \frac{l}{\epsilon},
$$

where $\epsilon$ is the UV cut off (lattice spacing).

The Rényi entanglement entropy, defined by

$$
S_{A}^{(n)}=\frac{1}{1-n} \log \operatorname{Tr}\left[\left(\rho_{A}\right)^{n}\right]
$$

can also be computed for the vacuum as

$$
S_{A}^{(n)}=\frac{c}{6}\left(1+\frac{1}{n}\right) \log \frac{l}{\epsilon}
$$




\subsection{EE for excited states from conformal transformation}

To calculate the EE for a special class of excited states in 2d CFTs, we can employ the conformal transformation. We transform the original coordinate system $(w, \bar{w})$ into a new one $(\xi, \bar{\xi})$ :

$$
\xi=f(w) .
$$

Also we need to note that the original UV cut off $\epsilon$, which is a lattice spacing in the $w$ coordinate, is mapped to the one, called $\tilde{\epsilon}_{a, b}$, in the new coordinate $(\xi, \bar{\xi})$ as

$$
\epsilon=\frac{\tilde{\epsilon}_{a}}{\left|f^{\prime}\left(w_{a}\right)\right|}=\frac{\tilde{\epsilon}_{b}}{\left|f^{\prime}\left(w_{b}\right)\right|} .
$$

Finally the EE for the excited state is found to be

$$
S_{A}=\frac{c}{6} \log \left[\frac{\left|f\left(w_{a}\right)-f\left(w_{b}\right)\right|^{2}}{\epsilon^{2}\left|f^{\prime}\left(w_{a}\right)\right|\left|f^{\prime}\left(w_{b}\right)\right|}\right] .
$$

In our analysis of joining/splitting local quenches, the EE is computed from a two point function of twist operators in the presence of a conformal boundary, called boundary conformal field theory (BCFT). Since two point functions in BCFT are similar to four point functions in CFTs without boundaries, we do not have any universal expression such as (2.3). However, we can obtain a definite analytical expression for special CFTs such as the Dirac fermion CFT and the holographic CFTs, whose details will be discussed later.

\subsection{Conformal map in $\mathrm{AdS}_{3} / \mathrm{CFT}_{2}$}

The AdS/CFT correspondence argues that gravitational theories on $\mathrm{AdS}_{3}$ is equivalent to $2 \mathrm{~d}$ holographic CFTs, which live on the AdS boundary [1]. The physical equivalence is formulated such that the partition function in each side agrees with the other one $[2,3]$, so called the bulk-boundary correspondence.

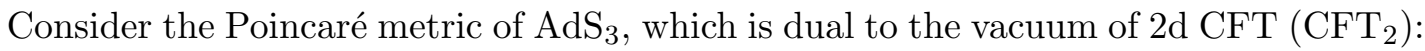

$$
d s^{2}=\frac{d \eta^{2}+d \xi d \bar{\xi}}{\eta^{2}}
$$

where we set the AdS radius to 1 for simplicity. The conformal transformation (2.8) is equivalent to the following coordinate transformation in $\mathrm{AdS}_{3}$ (see e.g. [35]):

$$
\begin{aligned}
& \xi=f(w)-\frac{2 z^{2}\left(f^{\prime}\right)^{2}\left(\bar{f}^{\prime \prime}\right)}{4\left|f^{\prime}\right|^{2}+z^{2}\left|f^{\prime \prime}\right|^{2}}, \\
& \bar{\xi}=\bar{f}(\bar{w})-\frac{2 z^{2}\left(\bar{f}^{\prime}\right)^{2}\left(f^{\prime \prime}\right)}{4\left|f^{\prime}\right|^{2}+z^{2}\left|f^{\prime \prime}\right|^{2}}, \\
& \eta=\frac{4 z\left(f^{\prime} \bar{f}^{\prime}\right)^{3 / 2}}{4\left|f^{\prime}\right|^{2}+z^{2}\left|f^{\prime \prime}\right|^{2}} .
\end{aligned}
$$

The metric in the coordinate $(w, \bar{w}, z)$ reads

$$
d s^{2}=\frac{d z^{2}}{z^{2}}+T(w)(d w)^{2}+\bar{T}(\bar{w})(d \bar{w})^{2}+\left(\frac{1}{z^{2}}+z^{2} T(w) \bar{T}(\bar{w})\right) d w d \bar{w},
$$


where

$$
T(w)=\frac{3\left(f^{\prime \prime}\right)^{2}-2 f^{\prime} f^{\prime \prime \prime}}{4 f^{\prime 2}}, \quad \bar{T}(\bar{w})=\frac{3\left(\bar{f}^{\prime \prime}\right)^{2}-2 \bar{f}^{\prime} \bar{f}^{\prime \prime \prime}}{4 \bar{f}^{\prime 2}},
$$

are the chiral and anti-chiral energy stress tensor.

\subsection{Holographic entanglement entropy in $\mathrm{AdS}_{3} / \mathrm{CFT}_{2}$}

In Euclidean setups, the holographic entanglement entropy (HEE) [36-38] is given by

$$
S_{A}=\frac{L}{4 G_{N}}
$$

in terms of the length $L$ of shortest geodesic which connects the two boundary points of the subsystem $A$. We also need to impose the homology constraint that this geodesic is homologous to the subsystem $A$ in the AdS geometry.

If we consider a connected geodesic which connects $w_{a}$ and $w_{b}$, then its length is given by

$$
L_{a b}=\log \frac{\left|\xi_{a}-\xi_{b}\right|^{2}}{\tilde{\epsilon}_{a} \tilde{\epsilon}_{b}}=\log \left[\frac{\left|f\left(w_{a}\right)-f\left(w_{b}\right)\right|^{2}}{\epsilon^{2}\left|f^{\prime}\left(w_{a}\right)\right|\left|f^{\prime}\left(w_{b}\right)\right|}\right],
$$

where note the relation (2.9), following from the above coordinate transformation around $\eta=0: \eta \simeq\left|f^{\prime}\right| z$. Then the HEE $\frac{L_{a b}}{4 G_{N}}=\frac{c}{6} L_{a b}$ reproduces the formula (2.10).

\subsection{EE from AdS/BCFT}

Since our coming analysis of joining/splitting local quenches require us to consider a CFT, let us extend the AdS/CFT to a setup where a holographic CFT is defined on a manifold $M$ with boundaries $\partial M$. In particular, when a linear combination of conformal symmetry is preserved on $\partial M$, we call it a boundary conformal field theory (BCFT). The holographic dual of a BCFT, called AdS/BCFT, can be constructed in the following way $[15,16]$ (see also [39] for an earlier argument). For a typical AdS/BCFT setup, refer to the left picture of figure 2. Consider a surface $Q$ which ends on $\partial M$ and extends into the bulk. We impose the following condition on $Q$

$$
K_{\mu \nu}-K h_{\mu \nu}=-T_{\mathrm{BCFT}} \cdot h_{\mu \nu}
$$

where $h_{\mu \nu}$ is the induced metric on $Q$ and $K_{\mu \nu}$ is the extrinsic curvature on $Q ; K$ is the trace $h^{\mu \nu} K_{\mu \nu}$. The constant $T_{\mathrm{BCFT}}$ describes the tension of the 'brane' $Q$ and can take both positive and negative values in general. This boundary condition (2.17) arises naturally in the AdS/BCFT setup as follows (for more detail refer to $[15,16]$ ). If we consider the standard gravity action given by the Einstein-Hilbert action plus the Gibbons-Hawking boundary term, it is well-known that there are two boundaries conditions: Dirichlet and Neumann. For AdS/BCFT, we choose the Neumann boundary condition as we want to keep the boundary $Q$ dynamical as is so in the BCFT boundary. The Neumann boundary condition is given by $K_{\mu \nu}-K h_{\mu \nu}=0$. To generalize this boundary condition, we add the tension term of the surface $Q$ to the bulk action, given by $T_{\mathrm{BCFT}} \int_{Q} \sqrt{h}$. This modified the Neumann boundary condition into the form (2.17). It is also useful to note that in explicit 
examples, we can confirm that this boundary condition (2.17) preserves the boundary conformal symmetry.

The gravity dual of a CFT on $M$ is given by the AdS gravity solution restricted on the space $N$, defined by the bulk region surrounded by $M$ and $Q$. To find such a solution, we need to solve the Einstein equation with the boundary condition (2.17), where the presence of $Q$ gives back-reactions and modifies the bulk metric [15, 16, 40, 41]. In our examples which we will discuss later, we can analytically find gravity duals of two dimensional BCFTs by using the bulk extension of the conformal map (2.12).

The region $N$ gets larger as the tension $T_{\mathrm{BCFT}}$ increases and this suggests that $T_{\mathrm{BCFT}}$ estimates the degrees of freedom on the boundary $\partial M$. Indeed, as shown in $[15,16]$ in the $\mathrm{AdS}_{3}$ case, the tension is monotonically related to the boundary entropy $S_{\text {bdy }}$ introduced in [42] as follows:

$$
S_{\mathrm{bdy}}=\frac{c}{6} \operatorname{arctanh}\left(T_{\mathrm{BCFT}}\right) .
$$

For notational simplicity, we introduce a positive parameter $k(>0)$ by

$$
S_{\text {bdy }} \equiv \frac{c}{12} \log k .
$$

A larger $k$ means a larger boundary entropy or tension $T_{\mathrm{BCFT}}$. In particular, we have $k=1$ for $T_{\mathrm{BCFT}}=S_{\mathrm{bdy}}=0$. Refer to e.g. [43-45] for studies of HEE in higher dimensional setups.

Later we will employ the AdS/BCFT to calculate the holographic entanglement entropy (HEE). Consider the holographic entanglement entropy $S_{A}$ for an interval $A$ in the $\mathrm{AdS}_{3} / \mathrm{BCFT}_{2}$ setup. As in the right picture, there are two possibility: connected one $S_{A}^{\text {con }}$ and the disconnected one $S_{A}^{\text {dis }}$. The latter arises because the geodesic which connects the two end points of $A$ can end on the boundary surface $Q$ in the middle as depicted in the right picture of figure 2 . The correct holographic entanglement entropy is given by the one with a smaller length.

The connected HEE $S_{A}^{\text {con }}$ is not affected by the boundary surface $Q$. Therefore, this simply agrees with the estimation (2.10) of the CFT without boundary. On the other hand, the disconnected HEE $S_{A}^{\text {dis }}$ is highly affected by the boundary $Q$ as the geodesic ends on $Q$. In the Poincare AdS setup, $S_{A}^{\text {dis }}$ in figure 2 is computed as (refer to $[15,16]$ for the derivation):

$$
S_{A}^{\mathrm{dis}}=\frac{c}{6} \log \left(\frac{2 s_{a}}{\tilde{\epsilon}_{a}}\right)+\frac{c}{6} \log \left(\frac{2 s_{b}}{\tilde{\epsilon}_{b}}\right)+2 S_{\mathrm{bdy}}
$$

where $s_{a, b}$ are the distance between the surface $Q$ and the two end points of the interval $A ; \tilde{\epsilon}_{a, b}$ are the UV cut off in the Poincare AdS at the two boundary points. Intuitively, as the surface $Q$ gets further from the subsystem $A$, then geodesic length (i.e. HEE) gets larger. Thus as the tensor $T_{\mathrm{BCFT}}$ gets larger, the HEE gets larger. This effect is universally described by the constant term proportional to $S_{\text {bdy }}$ in (2.20) as follows form the result in $[15,16]$. Note that this is the sum of two disconnected geodesics and therefore has the doubled contribution of $S_{\mathrm{bdy}}$.

We can also understand this from the CFT viewpoints. In the holographic CFTs (or large central charge CFTs with sparse spectrum). As in the standard large $c$ arguments [79], 

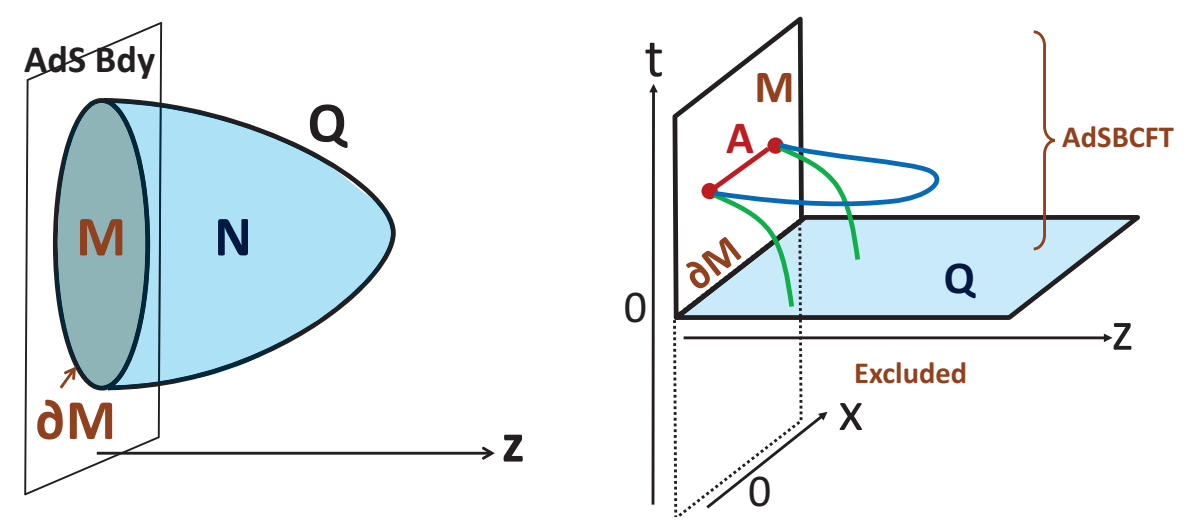

Figure 2. A sketch of AdS/BCFT analysis for $\mathrm{AdS}_{3}$. A holographic CFT on $M$ (with the boundary $\partial M$ ) is dual to gravity on $N$. The boundary of $N$ consists of the surface $Q$ and $M$. The right picture shows the calculation of holographic entanglement entropy. The blue curve gives the connected geodesic contribution and the green ones are the disconnected geodesics which end on the boundary surface $Q$. The correct holographic entanglement entropy is given by the one with a smaller length.

we can approximate a correlation function by a semiclassical saddle point. In our setup with a boundary, there are two saddles. One of them is obtained by contracting two twist operators, which give the connected EE $S_{A}^{\mathrm{con}}$. The other is given by contracting each of them with its mirror operator across the boundary, which leads to the disconnected one $S_{A}^{\text {dis }}$. Both of them agree with the results from AdS/BCFT. The $S_{\text {bdy }}$ dependence in $(2.20)$ occurs because the branch cut which extends from a twist operator ends on the boundary as is already known in the standard calculation of EE in BCFT [7, 8].

More generally, including higher dimensional setups, we can calculate the holographic entanglement entropy in the following way. In the standard holographic entanglement entropy without any boundaries, $S_{A}$ is given by the area of minimal or extremal surface $\Gamma_{A}$ which ends on $\partial A$ and which is homologous to $A$. In the presence of boundary surfaces $Q$, we impose the homology condition by regarding the surfaces $Q$ as trivial spaces with the zero size.

\section{Entanglement density}

Here we study a quantity called entanglement density (ED) introduced in [14]. First we would like to note that this is not a new quantity as it follows from the values of the entanglement entropy (EE). However, the ED provides a helpful way to display the behavior of EE in complicated systems, because we can capture the essence of time evolution from the behavior of ED as we explain later.

The ED is defined from the data of EE in a $2 \mathrm{~d}$ CFT when the subsystem $A$ is an interval. Since we have in mind generic excited states, we do not require the translational symmetry. When $A$ is given by an interval $a \leq x \leq b$ at a fixed time $t$, the EE is written as $S_{A}(a, b, t)$ (let us assume $a<b$ ). The entanglement density $n(a, b, t)$ is defined by

$$
n(a, b, t)=\frac{1}{2} \frac{\partial^{2} S_{A}(a, b, t)}{\partial a \partial b}=\frac{1}{2}\left(\frac{1}{4} \frac{\partial^{2}}{\partial \xi^{2}}-\frac{\partial^{2}}{\partial l^{2}}\right) S_{A}(\xi, l, t),
$$


where we introduced the center of the interval ${ }^{1} \xi$ and length $l$ such that

$$
a=\xi-\frac{l}{2}, \quad b=\xi+\frac{l}{2} .
$$

For example, the ED for a CFT vacuum, denoted by $n_{0}(\xi, l, t)$, takes the universal form:

$$
n_{0}(\xi, l, t)=\frac{c}{6 l^{2}} .
$$

By definition, the EE is represented as a double integral of ED:

$$
S_{A}(a, b, t)=\left(\int_{-\infty}^{a} d x \int_{a}^{b} d y+\int_{b}^{\infty} d y \int_{a}^{b} d x\right) n(x, y, t) .
$$

Therefore, if we assume all entanglement comes from the bipartite quantum correlation, the ED measures the number of EPR pairs between $x=a$ and $x=b$. However we should note that this interpretation is too naive as in general we have multi-partite entanglement in QFTs. This issues becomes serious in the later time behavior of holographic local operator quench [11], as pointed out in [46]. On the other hand, for the massless Dirac fermion CFT, this interpretation works so well that we can reproduce correct results from the universal ED (3.3) even when $A$ consists of multiple disconnected intervals as we will explain in the appendix A. One of the purposes of the present paper is to emphasize that the entanglement density at least provides a useful and simple way to extract the essential behaviors of the entanglement entropy in a systematical way. We will see this in many examples.

We are especially interested in the difference

$$
\Delta n(\xi, l, t)=n(\xi, l, t)-n_{0}(\xi, l, t),
$$

where $n_{0}(\xi, l, t)$ is given by (3.3). In other words, $\Delta n(\xi, l, t)$ is the entanglement density for the entanglement entropy growth

$$
\Delta S_{A}=S_{A}-S_{0 A}
$$

where $S_{0 A}$ is the entanglement entropy for the ground state.

\subsection{Properties}

As already noted in [14], the growth of $\operatorname{ED} \Delta n(\xi, l, t)$ enjoys several interesting properties. First of all, owing to the first law of entanglement entropy [47-49], in the small size limit $l \rightarrow 0$, we have

$$
\Delta n(\xi, 0, t)=-\frac{\pi}{3} T_{t t}(\xi, t)
$$

where $T_{t t}$ is the energy density.

Moreover, the growth of ED satisfies a sum rule: $\int d a \int d b \Delta n(a, b, t)=0$ if the total state is pure. In [14], this was proved when we impose the periodic boundary condition,

\footnotetext{
${ }^{1}$ In this paper, $\xi$ is also used to denote another quantity, the coordinate in AdS/BCFT given by (2.8) and (2.12). However, since the analysis of ED and discussions in AdS/BCFT do not appear at the same time, its meaning can be easily figured out from the context.
} 
which identifies $x=a$ with $x=b$. More generally, we can prove this even when the total space is an interval $0 \leq x \leq L$ as we will explain below. The precise statement of the sum rule is

$$
\int_{0}^{L} d b \int_{0}^{b} d a \Delta n(a, b, t)=0
$$

at any time $t$. To prove this, we can rewrite it as follows

$$
\int_{0}^{L} d b \int_{0}^{b} d a \frac{1}{2} \partial_{a} \partial_{b} \Delta S_{A}(a, b, t)=\int_{0}^{L} d b \frac{1}{2}\left[\left.\partial_{b} \Delta S_{A}(a, b, t)\right|_{a=b}-\left.\partial_{b} \Delta S_{A}(a, b, t)\right|_{a=0}\right] .
$$

First of all, the quantity $\left.\partial_{b} \Delta S_{A}\right|_{a=b}$ is vanishing because the first law (3.7) tells us the behavior $\Delta S_{A} \propto(b-a)^{2}$ when $|b-a|$ is very small. The second term in the right hand side also follows because

$$
\left.\int_{0}^{L} d b \partial_{b} \Delta S_{A}(a, b, t)\right|_{a=0}=\left.\Delta S_{A}(a, b, t)\right|_{b=L, a=0}-\left.\Delta S_{A}(a, b, t)\right|_{b=0, a=0}=0
$$

where we employed the first law behavior and the pure state property $S_{A}=S_{A^{C}}\left(A^{C}\right.$ is the complement of $A$ ).

It is also intriguing to note that we can also define the entanglement density for Rényi entanglement entropy (2.6). Since this quantity also has the property $\Delta S_{A}^{(n)} \propto(b-a)^{2}$ when $|b-a|$ is very small, the Rényi entanglement density $\Delta n^{(n)}(a, b, t)$ also satisfies the sum rule (3.8).

\subsection{Example 1: global quenches}

One of the simplest but non-trivial examples of homogeneous excited states in CFTs is the global quenches. This is triggered by a sudden change of the Hamiltonian from a gapped one to a critical one $H$ at a specific time $t=0$. As argued in [50], we can model this process by approximating the state just after the quench by a regularized boundary state $|\Psi(t=0)\rangle=e^{-\alpha H}|B\rangle$, where $\alpha$ is an infinitesimally small parameter of the regularization and $|B\rangle$ is a boundary state [51,52]. In general, The EE of a subsystem with length $l$ shows the linear growth and the saturation [50]

$$
\begin{array}{ll}
\Delta S_{A}=\frac{\pi c}{6 \alpha} t & (0<t \leq l / 2), \\
\Delta S_{A}=\frac{\pi c}{12 \alpha} l & (t>l / 2) .
\end{array}
$$

This linear growth can be explained by the entangled pair creations and their relativistic propagations [50].

In this case the ED looks like

$$
\Delta n(\xi, l, t)=\frac{\pi c}{24 \alpha} \delta(l-2 t)+\Delta n(\xi, l, t)_{U V} .
$$

The UV contribution $\Delta n(\xi, l, t)_{U V} \sim O\left(T_{t t}\right)$ is localized at short distances $l \leq \alpha$ such that it obeys the sum rule (3.8). 

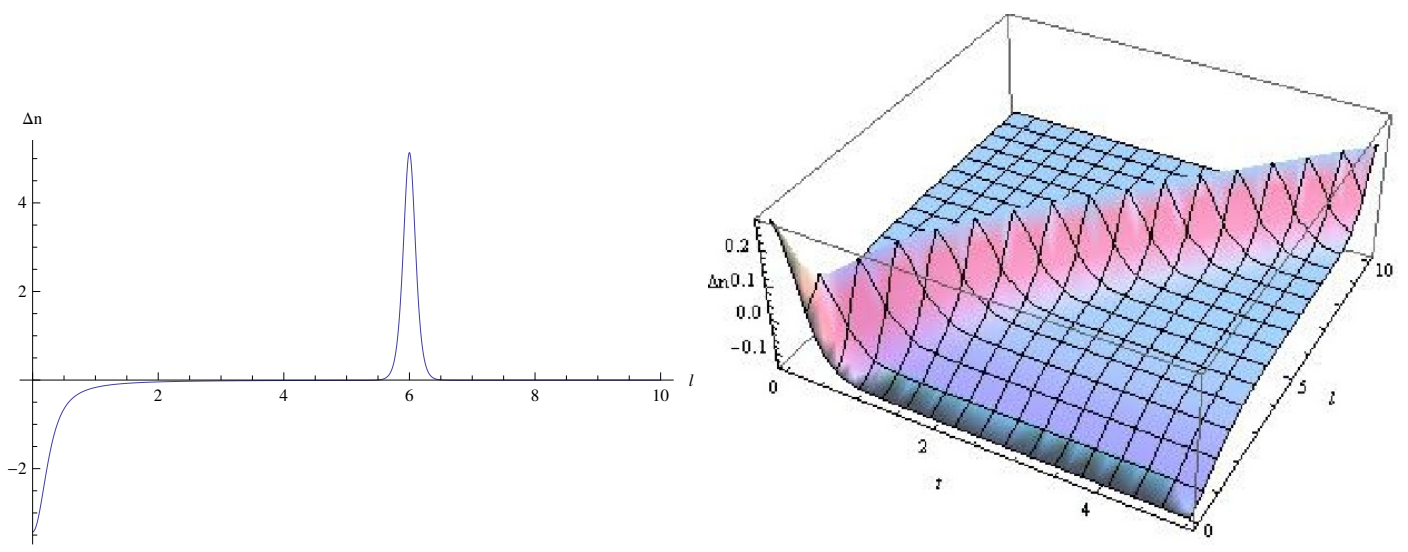

Figure 3. The behavior of entanglement density (ED) under the global quenches. The left graph describes the $\mathrm{ED} \Delta n(l, t)$ as a function of $l$ at $t=3$ (we chose $\alpha=0.1$ ). The right 3D plot shows the behavior of ED $\Delta n(l, t)$ as a function of the time $t$ (horizontal axis) and the subsystem size $l$ (depth axis), where we set $\alpha=0.5$.

If we consider the massless free Dirac fermion CFT in two dimension $(c=1)$ as a solvable example, we explicitly find the following expression of the $\mathrm{EE}^{2}$

$$
S_{A}=\frac{1}{6} \log \left[\frac{4 \alpha}{\pi \epsilon} \cdot \frac{\cosh ^{2}\left(\frac{\pi t}{2 \alpha}\right) \sinh ^{2}\left(\frac{\pi l}{4 \alpha}\right)}{\cosh \left(\frac{\pi}{2 \alpha}(t+l / 2)\right) \cosh \left(\frac{\pi}{2 \alpha}(t-l / 2)\right)}\right] .
$$

Thus, the ED reads

$$
\Delta n(l, t)=-\frac{1}{6 l^{2}}+\frac{\pi^{2}}{192 \alpha^{2}}\left(\frac{2}{\sinh ^{2}\left(\frac{\pi l}{4 \alpha}\right)}+\frac{1}{\cosh ^{2}\left(\frac{\pi}{2 \alpha}(t-l / 2)\right)}+\frac{1}{\cosh ^{2}\left(\frac{\pi}{2 \alpha}(t+l / 2)\right)}\right) .
$$

Indeed we can confirm the behavior (3.12) in this explicit example. We numerically plotted the profile of $\Delta n(l, t)$ in figure 3. We can also confirm that the sum rule $\int_{0}^{\infty} d l \Delta n(l, t)=0$, explicitly. Similar arguments can also be applied to holographic calculations of global quenches [54-57].

\subsection{Example 2: local operator quenches}

Next we consider the time evolution of an excited state produced by a local insertion of a primary operator $O$ at $x=0$ at the time $t=0[11,12]$ :

$$
|\Psi(t)\rangle=\mathcal{N}_{O} e^{-i H t} \cdot e^{-\alpha H} O(0)|0\rangle
$$

where $\alpha$ is again an infinitesimally small regularization parameter; $\mathcal{N}_{\mathcal{O}}$ is the normalization factor so that the state has the unit norm. We call its time evolution a local operator quench

\footnotetext{
${ }^{2}$ This is found by employing the conformal map $\xi=\exp \left(\frac{\pi w}{2 \epsilon}\right)$ and the expression (4.3) and (4.4). The same expression was obtained in [50]. For the boundary state we can choose either Dirichlet or Neumann boundary condition for the scalar field obtained from the bosonization, both of which lead to the same entanglement entropy by an appropriate choice of twist operators as shown in [53].
} 
(refer to the left picture of figure 1). We will discuss other types of local quenches (splitting and joining ones) in later sections, which are the main setups we consider in this paper. However, here we briefly discuss the operator quenches because they are instructive for our later arguments and the behaviors of their ED have not been studied well before.

Let us focus on two dimensional free CFTs or more generally rational CFTs (RCFTs) such as free scalar fields or minimal models, for simplicity. ${ }^{3}$ We again choose the subsystem $A$ to be the interval $a \leq x \leq b$. As found in [11, 12, 58], the evolution of (Rényi) EE looks like (in the limit $\alpha \rightarrow 0$ )

$$
\begin{aligned}
& \Delta S_{A}^{(n)}=0 \quad(0<t<|a|, \quad t>b) \\
& \Delta S_{A}^{(n)}=\log d_{O} \quad(|a|<t<b)
\end{aligned}
$$

for any $n$, including the $\operatorname{EE} n=1$. Here we assumed $b>|a|$. The quantity $d_{O}(\geq 1)$ is called the quantum dimension, which is a finite constant. This behavior is obvious from the picture that the operator insertion creates an entangled pair at $x=0$ and then it splits into the left and right moving quanta, which are entangled with each other as argued in $[11,12,58]$.

The entanglement density (ED) is found to be ${ }^{4}$

$$
\Delta n(a, b, t)=-\log d_{O} \cdot \operatorname{sgn}(a b) \cdot \delta(t-|a|) \delta(t-|b|)
$$

It is also straightforward to reproduce the behavior (3.16) by integrating this entanglement density over a suitable region of $(\xi, l)$ as we explain in the left and middle pictures of figure 4.

Let us confirm this in an explicit example of a massless free scalar field $\phi$. To obtain an analytical expression, we calculate the growth of 2nd Rényi entanglement entropy $\Delta S_{A}^{(2)}$. We choose the operator $O$ to be $O=e^{i \phi}+e^{-i \phi}$. In this case we have $d_{O}=2$ and $\Delta S_{A}=\log 2$ for $a<t<b$. This is simply because this operator creates the genuine Bell pair [11, 12, 58]. The explicit form of $\Delta S_{A}^{(2)}$ was obtained in [58] and is given by

$$
\Delta S_{A}^{(2)}=\log \left(\frac{2}{1+|z|+|1-z|}\right)
$$

where $z=\frac{\left(z_{1}-z_{2}\right)\left(z_{3}-z_{4}\right)}{\left(z_{1}-z_{3}\right)\left(z_{2}-z_{4}\right)}$ is the cross ratio of the four locations:

$$
z_{1}=-z_{3}=\sqrt{\frac{a-t-i \alpha}{b-t-i \alpha}}, \quad z_{2}=-z_{4}=\sqrt{\frac{a-t+i \alpha}{b-t+i \alpha}} .
$$

Indeed, in the limit $\alpha \rightarrow 0$, we can confirm $(z, \bar{z}) \rightarrow(0,0)$ for the regions $0<t<|a|$ and $t>b$, while we have $(z, \bar{z}) \rightarrow(1,0)$ when $|a|<t<b$. This leads to the result (3.16).

\footnotetext{
${ }^{3}$ Refer also to the papers [58-77] for further related calculations.

${ }^{4}$ To see this, note that for any $a, b$, we can write

$$
\Delta S_{A}=\log d_{O} \cdot(\theta(|b|-t) \theta(t-|a|)+\theta(t-|b|) \theta(|a|-t)),
$$
}

by using the Heaviside step function $\theta(x)$. 

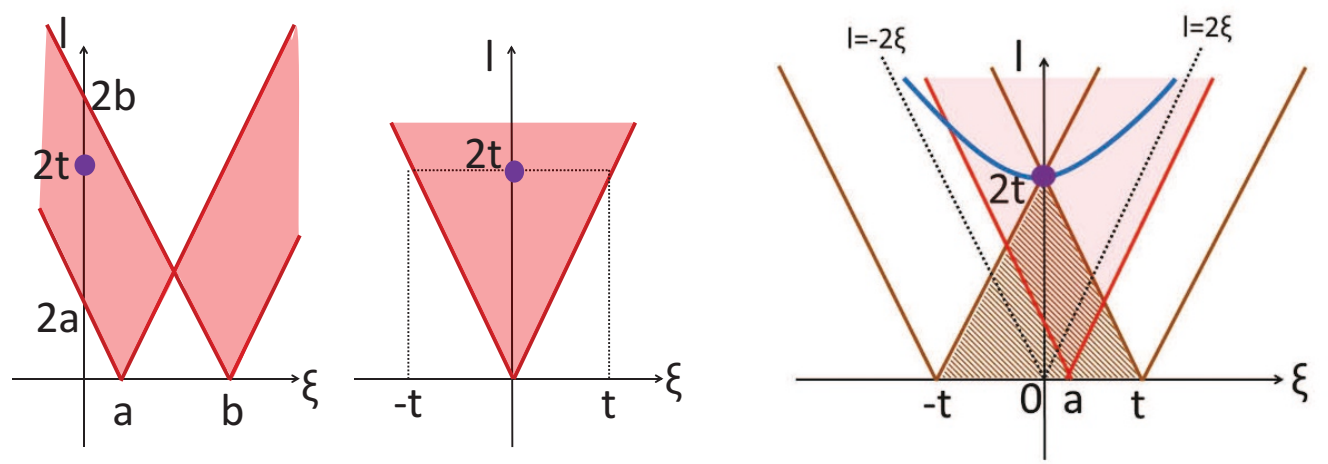

Figure 4. The integration range $\int d l d \xi$ of the ED (red regions) and causality range (brown shaded region). The left picture shows the region of integration which computes $S_{A}$ for the subsystems $A=[a, b]$. The middle one shows the same one when $A$ is the half line: $a=0$ and $b=\infty$. The brown shaded region in the right picture describe the range of $(\xi, l)$ where the local excitation at $x=t=0$ can make any physical influence assuming causality. In all these three pictures, the purple dot at $(\xi, l)=(0,2 t)$ represents the peak of ED due to the local quench. In the right picture, the blue curve near this dot (given by $l=2 \sqrt{\xi^{2}+t^{2}}$ ) describes the delta functional peaks which are peculiar to holographic local quenches. Note that they are outside of the causality region. The calculations of $S_{A}$ for $a \ll t \ll b$ correspond to integrating the red region in the right picture. We note the left part of the blue curve gradually gets into the red region, which gives the logarithmic growth of EE.
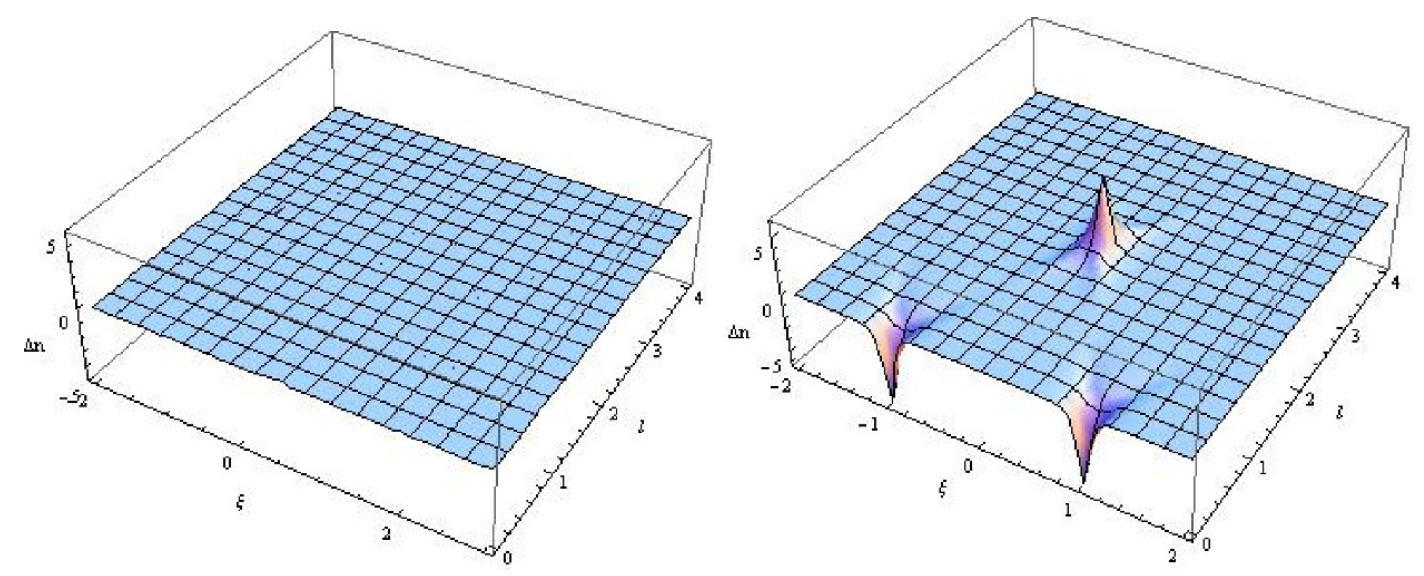

Figure 5. The profile of entanglement density for the 2nd Renyi entropy under the local operator quench in $c=1$ free scalar CFT at the time $t=0$ (left) and $t=1$ (right) with $\alpha=0.1$, trigger by the operator $O=e^{i \phi}+e^{-i \phi}$. The horizontal and depth coordinate correspond to $\xi$ and $l$, respectively.

We plotted the ED obtained from this Rényi entropy in figure 5 for $\alpha=0.1$. Indeed, we can numerically confirm the behavior (3.18) as well as the sum rule. It is also useful to note that the ED is vanishing completely at $t=0$ as the positive and negative delta-functional peaks coincide and cancel each other.

Holographic case. One may wonder if a similar result is true for local operator quenches in holographic CFTs. A holographic calculation for the operator local quench was given 
in [14] (see also [78]) and its entanglement entropy was reproduced from CFT analysis in [79]. Especially, the resulting entanglement entropy shows a logarithmic growth

$$
S_{A} \simeq \frac{c}{6} \log \frac{t}{\alpha}+\frac{c}{3} \log \frac{l}{\epsilon},
$$

when $|a| \ll t \ll b$. We will explain the geometric explanation in gravity dual in section 6 .

This logarithmic growth is clearly different from the previous RCFT case (3.16). This looks puzzling because the relativistic propagation picture leads to the behavior (3.16) and there seems to be no way to explain the slow logarithmic growth (3.21) in relativistic theories.

Interestingly, we can resolve this puzzle by looking at the ED instead of EE. Our argument here will be very brief because we will study this more closely in the joining local quench model, which has the similarity on this aspect. The plot of ED shows that the peak at $(\xi, l)=(2 t, 0)$ is not localized as opposed to the Dirac fermion case, but it is continuously distributed on a curve, which is depicted as the blue curve in the right picture of figure 4. We observe that the left part of peak curve (blue) enters into the integration region (red) as time evolves. Thus, we find that $S_{A}$ increases gradually under the time evolution. This qualitatively explains the behavior (3.21).

Note that the continuous peaks are outside of the region which we expect from the causality propagation of the local excitation inserted at $x=t=0$. The holographic analysis in [14] shows they are on the curve $l=2 \sqrt{\xi^{2}+t^{2}}$. In particular, at $t=0$, the peaks are distributed on $l=2|\xi|$. This is clear different from the RCFT examples, where the ED vanishes everywhere at $t=0$. From this, we learn that in holographic CFTs, the operator local quench (3.15) initially generates highly non-local entanglement, as opposed to what we expect from the naive causality argument. The relativistic propagation of this nonlocal entanglement is the reason why we find the logarithmic growth (3.21). This property is also expected from the fact that the insertion of local operator is not a local unitary transformation [67]. Note that this initial non-local entanglement can be negligible in the operator local quench in RCFTs, whose ED only has the localized peak at $(\xi, l)=(2 t, 0)$.

\section{Splitting local quench}

Now we move on to the main analysis of this paper. Consider the splitting process in a $2 \mathrm{~d}$ CFT. Namely, we start with a $2 \mathrm{~d}$ CFT on a connected line $-\infty<x<\infty$ and at $t=0$ we cut it into two halves at $x=0$, as sketched in the middle picture of figure 1 . The preparation of the quantum state at $t=0$ just after the splitting process can be done by considering the Euclidean path-integral as in figure 6 . The parameter $\alpha$ corresponds to the regularization of local quench. This setup is described by the conformal transformation:

$$
\xi=i \sqrt{\frac{w+i \alpha}{w-i \alpha}} \equiv f(w),
$$

which maps the Euclidean geometry with the cut: $-\alpha<\operatorname{Im}[w]<\alpha$ and $\operatorname{Re}[w]=0$ (the left picture of figure 6) into an upper half plane $\operatorname{Im}[\xi] \geq 0$. 

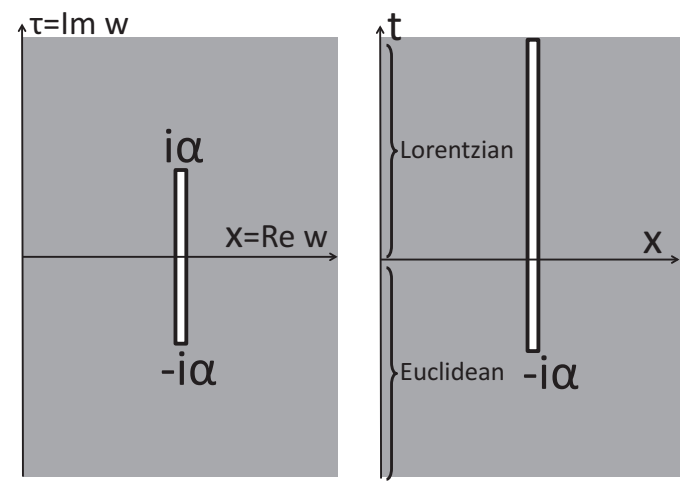

Figure 6. The geometries which realize the splitting local quenches. The left figure describes the space on which we perform the Euclidean path-integral. This is mapped into an upper half plane by the map (4.1). The right picture describes the path-integral realization of the time evolution after the split process happened at $t=0$, where the Euclidean path-integral for $\tau<0$ creates the state just after the splitting process.

\subsection{Splitting local quench in Dirac free fermion CFT}

One example where we can calculate the time evolution of EE under the splitting quench is the massless Dirac fermion CFT in two dimensions $(c=1)$. We choose the subsystem $A$ to be an interval $a \leq x \leq b$, or equally $l=b-a$ and $\xi=(a+b) / 2$. Due to the parity symmetry $x \rightarrow-x$, we can assume

$$
|a|<b, \quad b>0
$$

without losing generality. In the rest of this paper, we always focus on the entanglement entropy $S_{A}$ for this interval.

In the Dirac free fermion CFT, the twist operator $\sigma_{n}$ is described by a bosonization $\sigma_{n}=e^{\frac{i}{n} X}$, where $X$ is the free massless scalar field dual to the Dirac fermion $\psi$ via the standard relation $\psi=e^{i X}$. Since we know the analytical form of correlation functions of free scalar on an upper half plane, we can calculate the two point function of the twist operators even in the presence of the boundary as follows (this is identical to eq. (2.31) of $[18]$ see also $[9,10,53,80])$ :

$$
S_{A}=-\frac{1}{6} \log \left(F \epsilon^{2}\right)
$$

where $F$ is given by

$$
F=\left|\frac{d \xi_{a}}{d w_{a}}\right| \cdot\left|\frac{d \xi_{b}}{d w_{b}}\right| \cdot \frac{\left(\xi_{a}-\bar{\xi}_{b}\right)\left(\bar{\xi}_{a}-\xi_{b}\right)}{\left|\xi_{a}-\bar{\xi}_{a}\right|\left|\xi_{b}-\bar{\xi}_{b}\right|\left(\xi_{a}-\xi_{b}\right)\left(\bar{\xi}_{a}-\bar{\xi}_{b}\right)} .
$$

The analytical expressions of $S_{A}$ when $\alpha$ is infinitesimally small are given as follows. In the early time period, $0<t<|a|$, we have

$$
S_{A}=\frac{1}{3} \log (b-a) / \epsilon
$$



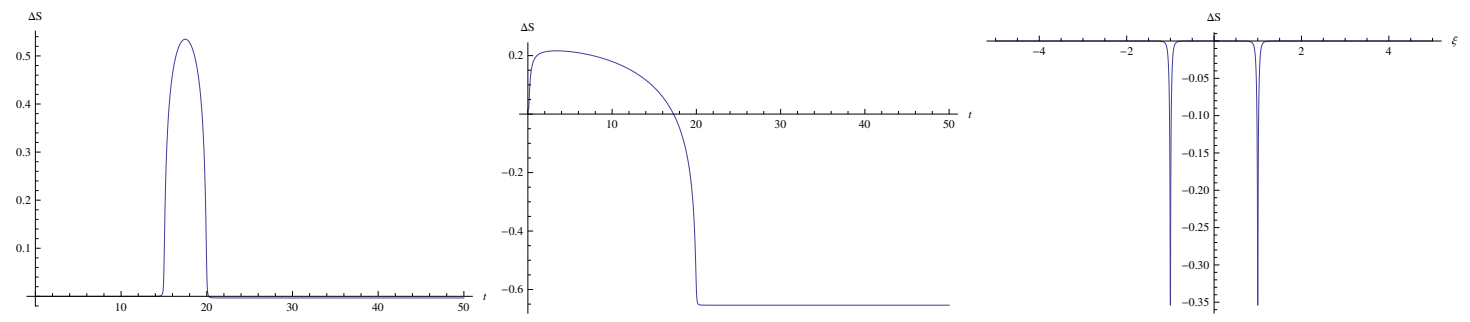

Figure 7. The plots of the entanglement entropy growth $S_{A}-S_{A}^{(0)}$ for $\alpha=0.1$. The left graph describes the time evolution when we take $(a, b)=(15,20)$. The middle one is the time evolution for $(a, b)=(0.1,20)$. The right one is the plot for various $\xi$ when we fix $l=2$ and $t=0$.

When $|a|<t<b$, we have

$$
S_{A}=\frac{1}{6} \log \frac{4|a|(b-a)(t-|a|)\left(b^{2}-t^{2}\right)}{(a+b)(t+|a|) \alpha \epsilon^{2}} .
$$

At late time, $t>b$, we have

$$
S_{A}=\frac{1}{6} \log \frac{4|a| b(b-|a|)^{2}}{(b+a)^{2} \epsilon^{2}}
$$

First, from (4.5), (4.6) and (4.7), we find that when the subsystem $A$ is symmetric around the origin i.e. $\xi=0$, the EE coincides with that for the ground state $S_{A}^{(0)}$ :

$$
S_{A}(0, l, t)=S_{A}^{(0)}=\frac{1}{3} \log \frac{l}{\epsilon} .
$$

We also plotted the numerical values of EE in figure 7 . When $\xi \neq 0$, we find $S_{A}-S_{A}^{(0)}$ gets non-trivially positive only for the period $|a|<t<b$. This agrees with the relativistic particle propagation picture of free massless Dirac fermion CFT. For $0<t<|a|$, the $\mathrm{EE}$ is the same as that for the vacuum. At the late time region $t>b$, it approaches to the EE for the ground state of the separated system. This is manifest in the left picture in figure 7. The middle picture in figure 7 shows that when we take $|a| \ll b$, the EE is gradually decreasing just after the sudden initial rise. Indeed, from (4.6), we find the analytical profile for $|a| \ll t<b$ :

$$
S_{A} \simeq \frac{1}{6} \log \frac{4|a|\left(b^{2}-t^{2}\right)}{\alpha \epsilon^{2}} .
$$

The right picture in figure 7 shows that the EE is reduced when either end point of $A$ gets closer to $x=0$, i.e. at the splitting point. This is because in this case, the EE only comes from that for a half-line.

The entanglement density (ED) can also be computed from the second differentiation of the EE (3.1). The results are plotted in figure 8. We can clearly see the positive peak at $(l, \xi)=(2 t, 0)$ as well as the negative peaks at $(l, \xi)=(0, \pm t)$, similarly to the local operator quench in RCFTs (3.18). The former agrees with the picture of propagating relativistic particles. The latter coincides the peaks of the energy density predicted from the first law of EE. In addition, there are also peaks near the origin $\xi=l=0$ and this is due to the cut 

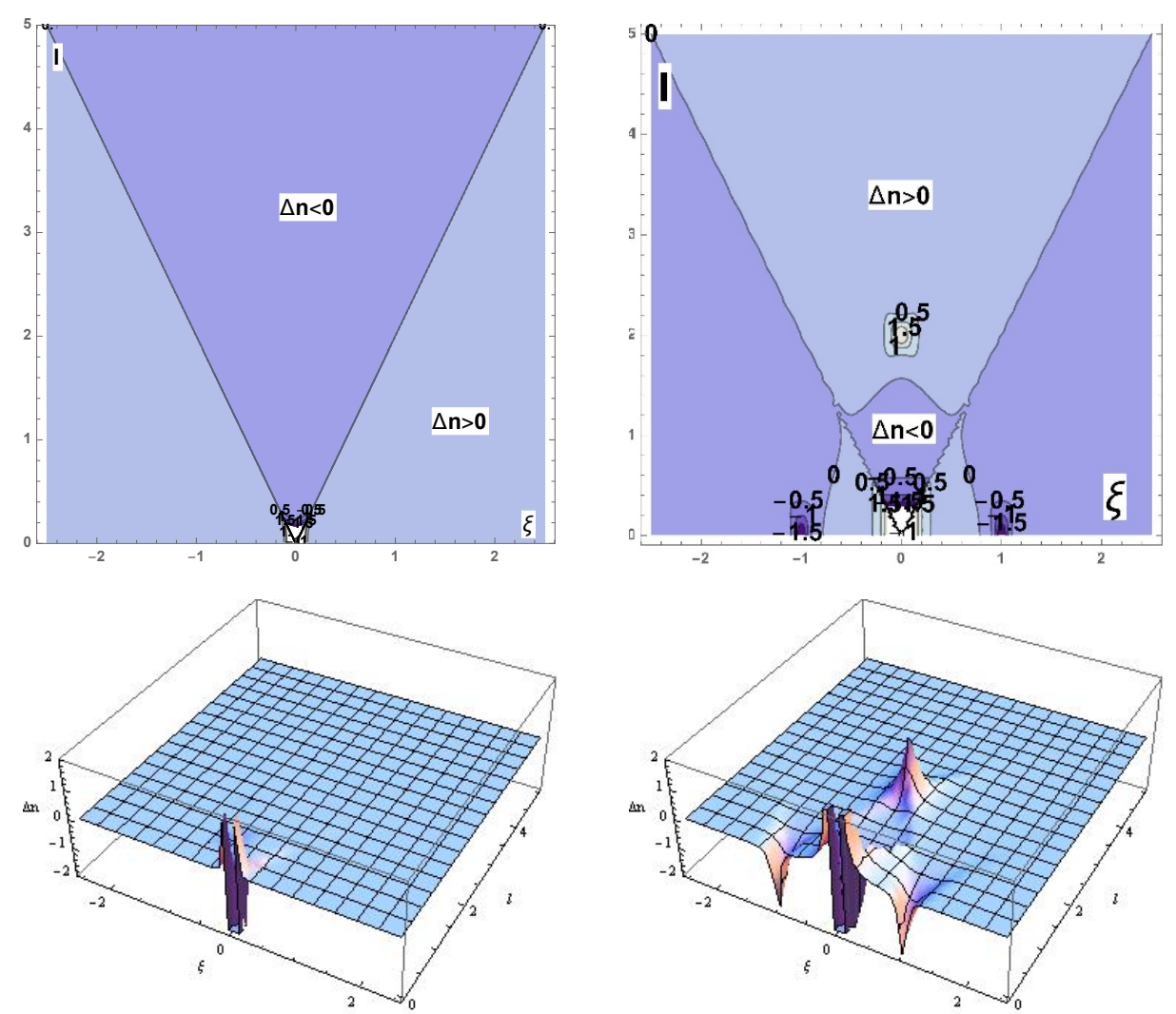

Figure 8. The plots of the entanglement density $\Delta n(\xi, l, t)$ for $\alpha=0.1$ at $t=0$ (left two graphs) and $t=1$ (right two graphs). The upper and lower graphs are the contour and 3D plots. The horizontal and depth coordinate corresponds to $\xi$ and $l$, respectively.

along $x=0$ where the space is divided into the left and right part, which is special to the splitting local quenches.

It is also interesting to note that a negative value region $\Delta n<0$ is expanding between these three peaks under the time evolution. This is responsible for the decreasing behavior of the $\mathrm{EE}$ (4.9) when we integrate the ED over the region given by the middle picture in figure 4.

\subsection{Splitting local quench in holographic CFT}

Another example which allows us to calculate the EE analytically is $2 \mathrm{~d}$ holographic CFTs. For the holographic CFT, we can apply the coordinate transformation (2.12) to calculate the EE. The dual Euclidean geometry is given by $\operatorname{Im}[\xi] \geq 0$ in the Poincaré AdS coordinate (2.11). The Lorentzian time evolution is obtained by performing the analytic continuation.

The holographic entanglement entropy (HEE) for the connected geodesic $S_{A}^{\text {con }}$ of the subsystem $A\left(=\left[w_{a}, w_{b}\right]\right)$ is computed by using (2.16) as follows:

$$
S_{A}^{\text {con }}=\frac{c}{6} \log \left[\frac{\left|f\left(w_{a}\right)-f\left(w_{b}\right)\right|^{2}}{\epsilon^{2}\left|f^{\prime}\left(w_{a}\right)\right|\left|f^{\prime}\left(w_{b}\right)\right|}\right]
$$

where $f(w)$ is given by (4.1). 
On the other hand, the HEE for the disconnected geodesics is found by applying the AdS/BCFT as we explained in eq. (2.20). The final result reads

$$
S_{A}^{\mathrm{dis}}=\frac{c}{6} \log \left(\frac{4\left(\operatorname{Im} f\left(w_{a}\right)\right)\left(\operatorname{Im} f\left(w_{b}\right)\right)}{\epsilon^{2}\left|f^{\prime}\left(w_{a}\right)\right|\left|f^{\prime}\left(w_{b}\right)\right|}\right)+2 S_{\mathrm{bdy}} .
$$

Note that the boundary entropy $S_{\text {bdy }}$ contribution arises because the location of boundary surface gets tilted according to the value of the tension $T_{\mathrm{BCFT}}$ following (2.18), so that it solves the boundary condition (2.17). The boundary entropy $S_{\text {bdy }}$ is parameterized by $k$ as in (2.19). Since $S^{\text {dis }}$ is the sum of two disconnected geodesic, we have the doubled contribution of $S_{\mathrm{bdy}}$ in (4.11). Refer to the right picture of figure 2 .

The final HEE is given by the smaller of the two:

$$
S_{A}=\min \left\{S_{A}^{\text {con }}, S_{A}^{\text {dis }}\right\} .
$$

The behaviors of EE are plotted in figure 9 by choose the vanishing tension $T_{\mathrm{BCFT}}=0$ or equally vanishing boundary entropy $S_{\mathrm{bdy}}=0$.

When $\alpha$ is infinitesimally small, we obtain the following analytical expressions (we take the boundary entropy arbitrary) as explained in the appendix B.

In the early time period, $0<t<|a|$, we have

$$
\begin{aligned}
S_{A}^{\mathrm{con}} & =\frac{c}{3} \log (b-a) / \epsilon \\
S_{A}^{\mathrm{dis}} & =\frac{c}{6} \log \frac{4\left(a^{2}-t^{2}\right)\left(b^{2}-t^{2}\right)}{\alpha^{2} \epsilon^{2}}+2 S_{\mathrm{bdy}} .
\end{aligned}
$$

When $|a|<t<b$, we have

$$
\begin{aligned}
& S_{A}^{\mathrm{con}}= \begin{cases}\frac{c}{6} \log \frac{2(b-a)(t-a)(b-t)}{\alpha \epsilon^{2}}, & (a>0) \\
\frac{c}{6} \log \frac{2(b-a)(t+a)(b+t)}{\alpha \epsilon^{2}}, & (a<0)\end{cases} \\
& S_{A}^{\mathrm{dis}}=\frac{c}{6} \log \frac{4|a|\left(b^{2}-t^{2}\right)}{\alpha \epsilon^{2}}+2 S_{\mathrm{bdy}} .
\end{aligned}
$$

At late time, $t>b$, we have

$$
\begin{aligned}
S_{A}^{\text {con }} & = \begin{cases}\frac{c}{3} \log (b-a) / \epsilon, & (a>0) \\
\frac{c}{6} \log \frac{4\left(t^{2}-a^{2}\right)\left(t^{2}-b^{2}\right)}{\alpha^{2} \epsilon^{2}}, & (a<0)\end{cases} \\
S_{A}^{\text {dis }} & =\frac{c}{6} \log \frac{4|a| b}{\epsilon^{2}}+2 S_{\mathrm{bdy}} .
\end{aligned}
$$

It is clear that at the late time limit $t \rightarrow \infty$, the EE approaches to a constant value

$$
S_{A}(t \rightarrow \infty)=\min \left[\frac{c}{3} \log \frac{b-a}{\epsilon}, \frac{c}{6} \log \frac{4|a| b}{\epsilon^{2}}+2 S_{\mathrm{bdy}}\right],
$$

which agrees with the expected result for the separated two half lines. 

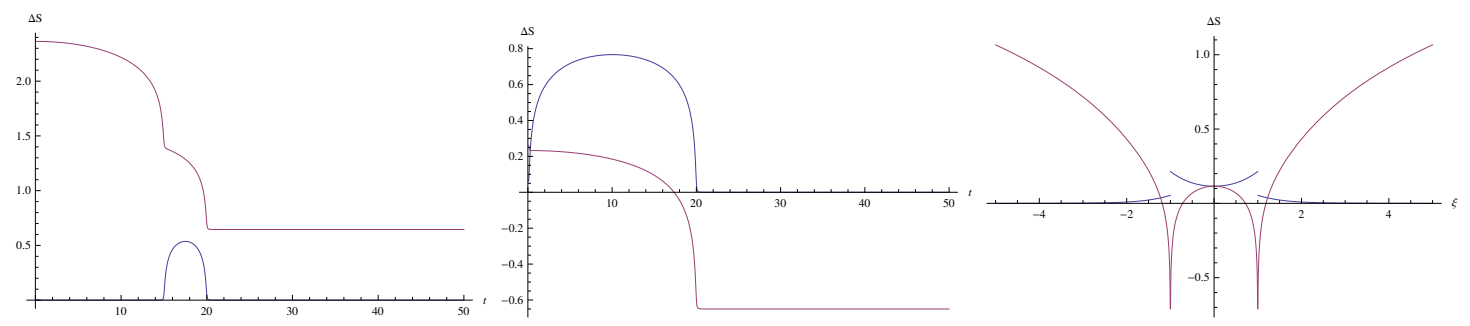

Figure 9. The plots of the holographic entanglement entropy growth $S_{A}-S_{A}^{(0)}$ at $\alpha=0.1$. We assume the tension of the boundary surface $Q$ is vanishing $T_{\mathrm{BCFT}}=0$. The blue/red graph describes the connected/disconnected contribution. The left graph describes the time evolution when we take $(a, b)=(15,20)$. The middle one is the time evolution for $(a, b)=(0.1,20)$. The right one is the plot for various $\xi$ when we fix $l=2$ and $t=0$.

The behavior of entanglement entropy is also numerically plotted in figure 9 by choosing the vanishing tension $T_{\mathrm{BCFT}}=0$ or equally vanishing boundary entropy $S_{\mathrm{bdy}}=0$. The left graph looks very similar to the one in the Dirac fermion case, which is interpreted by the propagation of relativistic particles. However, it is intriguing to note that if the boundary entropy $S_{\text {bdy }}$ is positive and very large, then $S_{A}^{\text {con }}$ can dominate in some region and gives a qualitative discrepancy from the Dirac fermion result.

It is interesting to ask the time evolution of $\mathrm{EE}$ when we choose the subsystem $A$ to be almost a half of the total system i.e. $a \ll t \ll b$. If we choose the boundary entropy $S_{\text {bdy }}$ is very large such that $k \gg 1$ (remember the definition of $k(2.19)$ ), then $S_{A}^{\text {con }}$ is favored for the period $a \ll t \ll(2 k+1) a$, which results in the logarithmic growth

$$
S_{A}^{\text {con }} \simeq \frac{c}{6} \log \frac{2 t}{\alpha}+\frac{c}{3} \log \frac{l}{\epsilon} .
$$

This is peculiar to the holographic CFTs. However, if $k$ is order one, this logarithmic growth is missing and the EE monotonically decreases as in the previous Dirac fermion example.

The right picture (assuming $S_{\mathrm{bdy}}=0$ ) in figure 9 is qualitatively similar to what we obtained for the free Dirac fermion CFT. For finite $\alpha>0$, one may notice that the entanglement entropy for the connected curve $S_{A}^{\text {con }}$ is not continuous as in the third graph in figure 9. However, this occurs when one of the endpoints of the subsystem $A$ coincides with $x=0$ and thus this discontinuity should happen because the left and right at $x=0$ are disconnected in the splitting quench. This can be easily understood if we go to the Poincaré coordinate (2.11).

The entanglement density is plotted in figure 10. In the upper graphs, we smeared the derivatives in (3.1) by replacing it with the finite difference: e.g. $\partial_{a} S(a, b, t) \rightarrow(S(a+$ $\delta / 2, b, t)-S(a-\delta / 2, b, t)) / \delta$. We did so because in this holographic case, we expect genuine delta-functional behaviors due to the phase transitions of HEE. In addition to the expected positive peak at $(\xi, l)=(0,2 t)$ and the two negative ones at $(\xi, l)=( \pm t, 0)$, we observe continuous peaks along the following two curves

$$
\begin{aligned}
& \text { Curve } 1: l=-t+\frac{|\xi|}{1+k}+\sqrt{\left(t-\frac{|\xi|}{1+k}\right)^{2}+\frac{4 k}{k+1}(t+|\xi|)|\xi|} \\
& \text { Curve } 2: l=t+\frac{|\xi|}{1+k}+\sqrt{\left(t+\frac{|\xi|}{1+k}\right)^{2}-\frac{4 k}{k+1}(t-|\xi|)|\xi|}
\end{aligned}
$$


where we used the positive parameter $k$ defined in (2.19). These two curves are located at phase transition points between $S^{\text {con }}$ and $S^{\text {dis }}$ as we can see from (4.14), where (4.18) and (4.19) are situated in $a>0$ and $a<0$, respectively. Their profile is plotted in the lower left picture of figure 10. Both of them coincides with $l=2|\xi|$ at $t=0$. In the limits $t \rightarrow \infty$ and $|\xi| \rightarrow \infty$, the curves are approximated by

$$
\begin{aligned}
& \text { Curve 1 : } \quad l \simeq \frac{2 k}{k+1}|\xi|, \quad(t \rightarrow \infty), \quad l \simeq 2|\xi|-\frac{2 t}{2 k+1}, \quad(|\xi| \rightarrow \infty) \\
& \text { Curve 2: } \quad l \simeq 2 t-\frac{2(k-1)}{k+1}|\xi|, \quad(t \rightarrow \infty), \quad l \simeq 2|\xi|+\frac{2 t}{2 k+1} . \quad(|\xi| \rightarrow \infty)
\end{aligned}
$$

The presence of these peak curves is peculiar to holographic CFTs and is missing in the free Dirac fermion CFTs or more generally RCFTs. They are outside of the causality zone and therefore the initial state already has highly non-local entanglement. In the lower right picture of figure 10, we sketched the calculation of EE, which is given by the integration of ED over the red region. For this, we notice that the blue curve (4.19) gets into the red region, while the green curve (4.18) goes out. Thus, we can explain the absence of the logarithmic growth of EE for the splitting local quenches due to the cancellations between these two opposite effects. ${ }^{5}$ Also we will provide the geometric explanation of the $\log t$ behaviors in section 6 .

\subsection{Holographic geometry of splitting local quench}

Now let us study the holographic geometry dual to the splitting local quench. The metric of the gravity dual is given by (2.13) with

$$
T(w)=\frac{3 \alpha^{2}}{4\left(w^{2}+\alpha^{2}\right)^{2}}, \quad \bar{T}(\bar{w})=\frac{3 \alpha^{2}}{4\left(\bar{w}^{2}+\alpha^{2}\right)^{2}},
$$

where $w=x+i \tau$ and $\bar{w}=x-i \tau$. For its Lorentzian extension, we can set $w=x-t$ and $\bar{w}=x+t$. The important ingredient of AdS/BCFT as reviewed in section 2.5 is the boundary surface $Q$ which extends from the AdS boundary $z=0$ to the bulk AdS. At $z=0, Q$ coincides with the cut in figure 6 , which describes the splitting process in the $2 \mathrm{~d}$ CFT. Therefore, we have to be careful in its global geometry i.e. which part of the geometry we should pick up and where the boundary surface $Q$ in the AdS/BCFT prescription is located.

Let us consider the Euclidean geometry dual to the left CFT picture of figure 6. It is important to note that the gravity dual is precisely defined by mapping an upper half $\operatorname{Im}[\xi] \geq 0$ of the Poincaré $\mathrm{AdS}_{3}$ (2.11) using the transformation (2.12) with (4.1), assuming that the tension is vanishing $T_{\mathrm{BCFT}}=0$. The boundary $Q$ in the AdS/BCFT, given by $\operatorname{Im}[\xi]=0$ in the latter setup, is mapped to a region on $x=0$.

First we focus on the time slice $\tau=0$ (i.e. $\operatorname{Im}[w]=0$ ) of the gravity dual. The metric on this time slice is given by

$$
d s^{2}=\frac{d z^{2}}{z^{2}}+\left(\frac{1}{z}+\frac{3 \alpha^{2} z}{4\left(\alpha^{2}+x^{2}\right)^{2}}\right)^{2} d x^{2}
$$

\footnotetext{
${ }^{5}$ However, if we consider the case $k \gg 1$, then (4.18) approaches $l=2 \xi$ and thus this curve is fully included in the red region until $t$ gets very large. This explains the logarithmic growth (4.17), peculiar to the large $k$ case.
} 

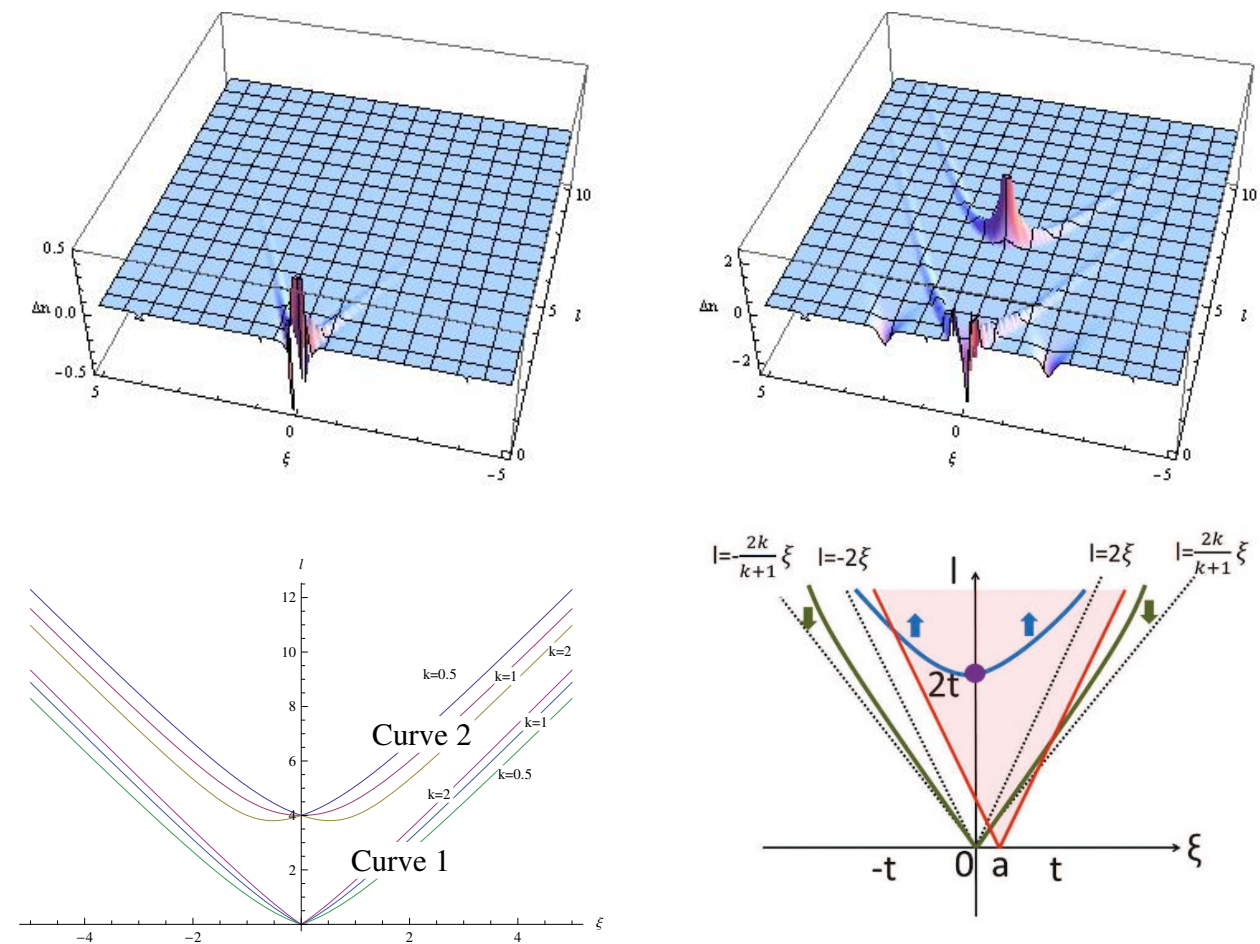

Figure 10. The behaviors of a smeared entanglement density (ED) for the holographic splitting local quench. The upper left and right graph are the plots of $\Delta n(\xi, l, t)$ at $t=0$ and $t=2$, respectively with $\alpha=0.1$ (again we set $T_{\mathrm{BCFT}}=0$ ). The horizontal and depth coordinate corresponds to $\xi$ and $l$, respectively. Here we smeared the originally delta functional behavior of the ED by replacing the second derivatives w.r.t. $l$ and $\xi$ with finite-differences $\delta=0.2$. We observe delta functional behaviors on the two curves (4.18) and (4.19) at $k=1$. The lower left picture shows these two curves at $t=2$ for $k=0.5,1,2$. The lower right picture describes the calculation of $S_{A}$ for $a \ll t \ll b$ by integrating the ED over the red region. The green and blue curves represent curve 1: (4.18) and curve 2: (4.19). We find that the blue curve gets into the red region, while the green curve goes out. The cancellations between them leads to the absence of logarithmic growth.

We can confirm that this slice is mapped into a quarter of the sphere given by $|\xi|^{2}+\eta^{2}=$ 1 with $\eta \geq 0$ and $\operatorname{Im}[\xi] \geq 0$. However, to realize one to one map, we need to remove the region

$$
z>\frac{2\left(x^{2}+\alpha^{2}\right)}{\alpha}
$$

and identify $x$ with $-x$ for any $x$ along the curve $z=\frac{2\left(x^{2}+\alpha^{2}\right)}{\alpha}$. A sketch of the map (2.12) at $t=0$ is depicted in figure 11. The boundary surface $Q$ extends from the AdS boundary $z=0$ toward the IR region but it ends at $z=2 \alpha$ due to the identification. This shows that the two half lines $x>0$ and $x<0$ at the AdS boundary $z=0$, is connected in the bulk through the horizon given by the identified curve $z=\frac{2\left(x^{2}+\alpha^{2}\right)}{\alpha}$. Therefore the CFTs on these two half lines are entangled and the entanglement entropy is estimated as the length of this curve:

$$
S_{A}=\frac{c}{6} \int_{0}^{x_{\infty}} \frac{2 d x}{\sqrt{x^{2}+\alpha^{2}}} \simeq \frac{c}{6} \log \frac{z_{\infty}}{\alpha}
$$




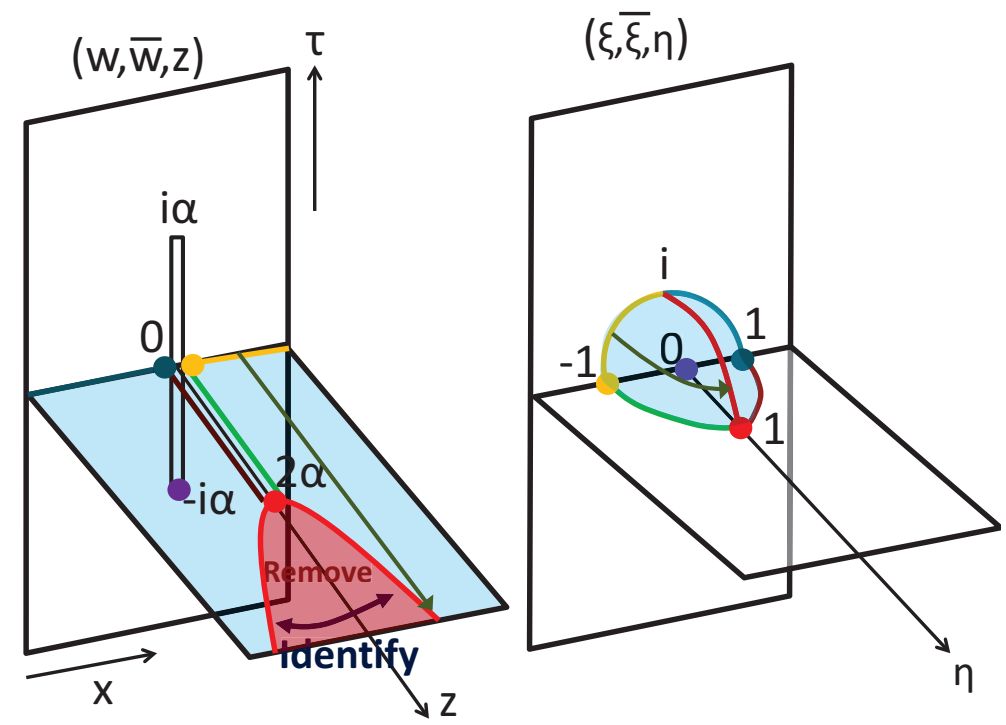

Figure 11. The map (2.12) between the gravity dual of splitting local quench (left) and the upper Poincaré AdS (right). The red curve in the left is given by $z=\frac{2\left(x^{2}+\alpha^{2}\right)}{\alpha}$ and the region inside this curve should be removed with the identification $x$ with $-x$ on the curve. The length of red curve gives the HEE between two separated lines. The green and dark brown curve describe the boundary surface $Q$.

where $z_{\infty}=2 x_{\infty}^{2} / \alpha$ is the IR cut off. This agrees with our expectation because the state before the splitting of the system was the ground state of the CFT on a line. Note that in the Poincaré coordinate, this is equal to a quarter of the largest circle in the sphere $|\xi|^{2}+\eta^{2}=1$, depicted as the red curve in the left picture of figure 11 .

Next we would like to examine how the boundary $Q$ in the AdS/BCFT looks like in our gravity dual. To see this we analyze which region is mapped into $\operatorname{Im}[\xi]=0$. It is straightforward to see that the boundary $Q$ should be on the slice $x=0$ (i.e. $\operatorname{Re}[w]=0$ ). Notice that we need to distinguish the two segments $Q_{+}$and $Q_{-}$of the boundary surface $Q$ at $x=+\delta$ and $x=-\delta(\delta>0$ is infinitesimal $)$, which corresponds to $\operatorname{Re}[\xi]<0$ and $\operatorname{Re}[\xi]>0$ in the Poincaré AdS. We focus on the time period $-\pi \alpha<\tau<\pi \alpha$. In figure 12, we sketched how the boundary surface $Q_{+}$looks like in the $(w, \bar{w}, z)$ coordinate and how it is mapped into the boundary $\operatorname{Im}[\xi]=0$ and $\operatorname{Re}[\xi]<0$ in the Poincaré coordinate for $x=+\delta$.

The tip $z=2 \alpha$ of the identification region (4.22) at the time slice $\tau=0$ is extended along

$$
z=\frac{2\left(\alpha^{2}-\tau^{2}\right)}{\sqrt{\alpha^{2}-4 \tau^{2}}}
$$

for $-\frac{\alpha}{2}<\tau<\frac{\alpha}{2}$. This is the red curve in figure 12. Note that this tip is the end point of the boundary surface $Q$ in the case of $T_{\mathrm{BCFT}}=0$. Therefore the two sheets $(x=+\delta$ and $x=-\delta$ ) are identified along this curve (4.24) with each other.

There is one more non-trivial issue. To secure one to one mapping into the half of Poincaré AdS, we need to remove the white region in the left picture in figure 12 and join the two blue curves with each other such that it agrees with the right picture i.e. $\operatorname{Im}[\xi]=0$ 

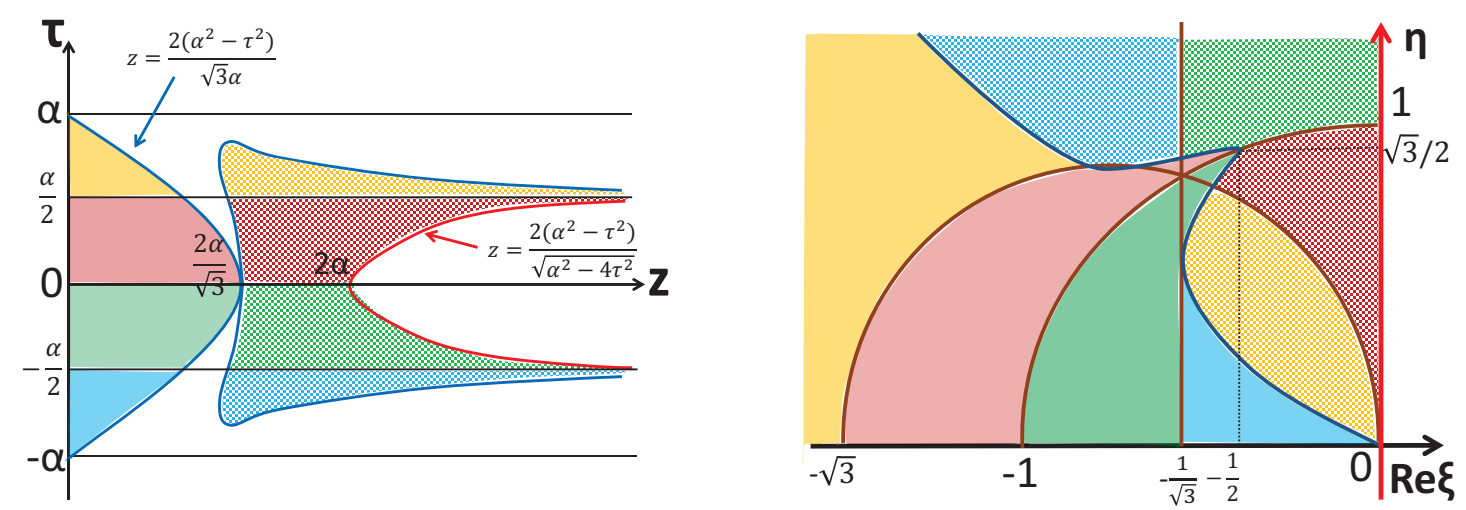

Figure 12. The boundary surface $Q_{+}$for $T_{\mathrm{BCFT}}=0$ in the gravity dual of splitting local quench. The left picture describes in the coordinate $(w, \bar{w}, z)$ setting $x=+\delta \rightarrow 0$. The right picture does in the Poincaré coordinate $(\xi, \bar{\xi}, \eta)$ on the boundary $\operatorname{Im}[\xi]=0$. The red curve in the left picture is mapped into the $\operatorname{Re}[\xi]=0$ in the right one. The two blue curves in the left are pasted with each other in a way they are mapped into the blue curve in the right picture. The eight colored regions in the left are mapped into those in the right. Note that there are one more boundary surface corresponding to $x=-\delta \rightarrow 0$, which is given by the same structure and is mapped to the $\operatorname{Re}[\xi]>0$ region. The white region in $-\frac{\alpha}{2}<\tau<\frac{\alpha}{2}$ should also be removed to secure the map is one to one.

in the Poincaré coordinate. Finally we can confirm that the colored regions in the left picture are mapped into those in the right one in figure 12. The full boundary surface $Q$ is given by joining two copies of such a space.

We will not get into details of the geometry in other parts as we can understand how the Lorentzian space looks like from the above observations. Nevertheless, it is helpful to point out that the identification surface (4.24) at non-zero $x$ is found to be

$$
z=\frac{2 \sqrt{x^{2}+(\alpha+\tau)^{2}} \sqrt{x^{2}+(\alpha-\tau)^{2}}}{\sqrt{\alpha^{2}-\left(\sqrt{x^{2}+(\alpha+\tau)^{2}}-\sqrt{x^{2}+(\alpha-\tau)^{2}}\right)^{2}}} \equiv G_{E}(x, \tau) .
$$

In the Lorentzian signature, this surface looks like

$$
z=\frac{2 \sqrt{x^{2}+(\alpha+i t)^{2}} \sqrt{x^{2}+(\alpha-i t)^{2}}}{\sqrt{\alpha^{2}-\left(\sqrt{x^{2}+(\alpha+i t)^{2}}-\sqrt{x^{2}+(\alpha-i t)^{2}}\right)^{2}}} \equiv G_{L}(x, t) .
$$

Thus the Lorentzian geometry for $t>0$ in the $(w, \bar{w}, z)$ is given by removing the part $z>G_{L}(x, t)$ and by identifying each two points on $(4.26)$ as $(\tau, x, z) \sim(\tau,-x, z)$. The boundary surface $Q$ consist of identical two surfaces $Q_{+}$and $Q_{-}$which extend in $z<$ $\frac{2\left(\alpha^{2}+t^{2}\right)}{\sqrt{\alpha^{2}+4 t^{2}}}$ and are localized at $x=\delta$ and $x=-\delta$, respectively. The final spacetime of the gravity dual is depicted in figure 13 .

At late time we can approximate this as

$$
z \simeq t .
$$

This behavior $z \sim t$ at $x=0$ clearly shows that the two half lines at the boundary are not causally connected in a marginal sense. Therefore the region $x>0$ and $x<0$, which 


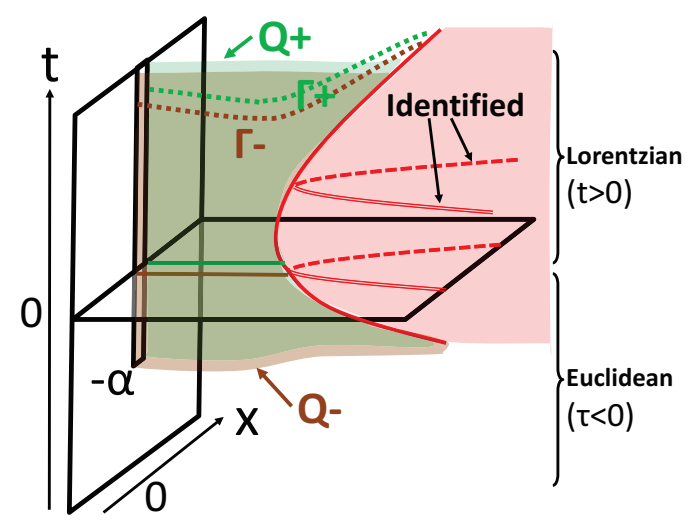

Figure 13. The spacetime geometry of gravity dual of the splitting local quench. The green surface $\left(Q_{+}\right)$and brown one $\left(Q_{-}\right)$are the boundary surface $Q$ in the AdS/BCFT. The red region defined by $z>G_{L}(x, t)$ should be removed with the dotted red curve and the doubled red curve identified. Thus this identified red region corresponds to the horizon. The region $x>0$ and $x<0$, which are separated at the AdS boundary $z=0$ are connected (in a space-like way) through this horizon in the bulk.

are separated at the AdS boundary $z=0$ are connected in a space-like way (i.e. it is a non-traversable wormhole) through this horizon in the bulk. This is consistent with the fact that in the CFT side there is no direct interaction between these two CFTs for $t>0$, though they are entangled.

As in our arguments in this subsection we assumed $T_{\mathrm{BCFT}}=0$ for simplicity, we would like to briefly mention how the gravity dual is modified when $T_{\mathrm{BCFT}} \neq 0$. When $T_{\mathrm{BCFT}}$ is non-zero, the boundary surface $Q$ in $(\xi, \bar{\xi}, \eta)$ will be tilted as

$$
\operatorname{Im}[\xi]=-\frac{T_{\mathrm{BCFT}}}{\sqrt{1-T_{\mathrm{BCFT}}^{2}}} \eta .
$$

When $T_{\mathrm{BCFT}}<0$, the boundary surfaces $Q_{+}$and $Q_{-}$tilted in the bulk such that they still coincide at $z=0$ and the angle between them takes a fixed positive value. Therefore, the bulk region gets squeezed and the entanglement entropy $S_{A}(4.23)$ is decreased. When $T_{\mathrm{BCFT}}>0$, the boundary surfaces are modified in an opposite way and the bulk region expands. The entanglement entropy $S_{A}$ gets increased.

In this subsection we studied the Lorentzian motion of the boundary surface $Q$. For the holographic entanglement entropy, we actually need the profile of geodesic in Lorentzian geometry, though in the previous subsection we employed the formal Wick rotation arguments to avoid this analysis. We will explicitly study how the Lorentzian geodesic looks like in section 6 .

\subsection{Splitting local quench in a simple spin system}

Before we go on, we would like to present numerical results for a splitting local quench in a simple spin model for a reference. We start with a spin- $1 / 2$ transverse Ising chain with $L$ sites and free boundary condition. Cut the interaction between site $j$ and $j+1$ to separate 


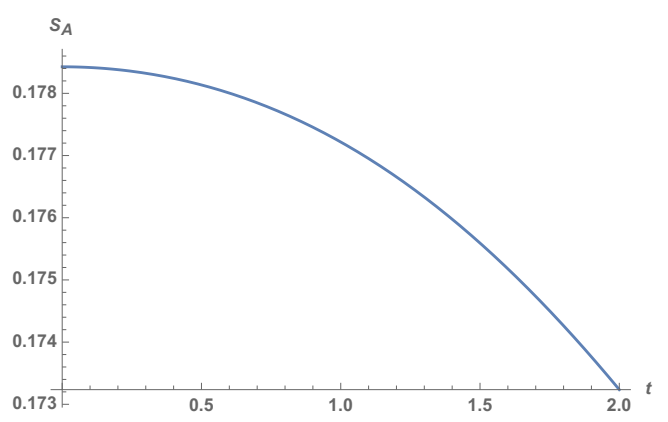

(a) $0 \leq t \leq 2$

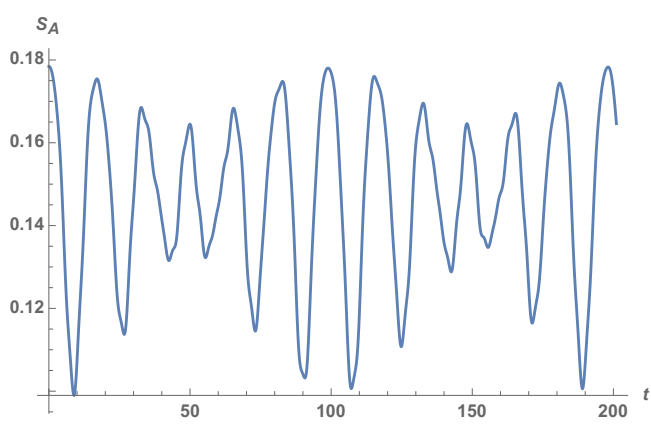

(b) $0 \leq t \leq 64 \pi$

Figure 14. Splitting local quench in spin-1/2 transverse Ising model: time evolution of EE $S_{A}$ for $J=h=1, L=6$, and $j=3$, where the subsystem $A$ is chosen to be $A=\{i \mid i=4,5\}$. We can see oscillation in long time range, while the short-time behavior of $S_{A}$ is very similar to those in CFT cases.

it into two independent chains at $t=0$. In this case Hamiltonian before quench $H_{\text {before }}$ and that after quench $H_{\text {after }}$ turns out to be

$$
\begin{aligned}
H_{\text {before }} & =-J \sum_{i=1}^{L-1} \sigma_{i}^{z} \sigma_{i+1}^{z}+h \sum_{i=1}^{L} \sigma_{i}^{x} \\
H_{\mathrm{after}} & =-J\left(\sum_{i=1}^{L-1} \sigma_{i}^{z} \sigma_{i+1}^{z}-\sigma_{j}^{z} \sigma_{j+1}^{z}\right)+h \sum_{i=1}^{L} \sigma_{i}^{x}
\end{aligned}
$$

Then let us start with the ground state of $H_{\text {before }}$ and investigate its dynamics under $H_{\text {after }}$ at $t \geq 0$. We presented our numerical results in figure 14 . We can see the behavior of its EE at an early time is very similar to the result in free fermion (4.6) and the disconnected contribution in holographic CFTs (4.14), qualitatively. At late time we observe oscillatory behavior because our spin system has a finite size.

\section{$5 \quad$ Joining local quench}

Consider a 2d CFT on two semi-infinite lines $x>0$ and $x<0$. We join each endpoint $x=0$ at $t=0$ as in the right picture of figure 1. This setup is described by the path-integral depicted in figure 15. We can map the Euclidean space (the left picture) into an upper half plane by the conformal map:

$$
\xi=i \sqrt{\frac{i \alpha-w}{i \alpha+w}} \equiv f(w) .
$$

The parameter $\alpha$ again corresponds to the regularization of local quench. We choose the subsystem $A$ to be $a \leq x \leq b$ at time $t$ as before. This corresponds to

$$
w_{a}=a-t, \quad \bar{w}_{a}=a+t, \quad w_{b}=b-t, \quad \bar{w}_{b}=b+t,
$$

where we performed the analytical continuation of the Euclidean time $\tau=i t$. 

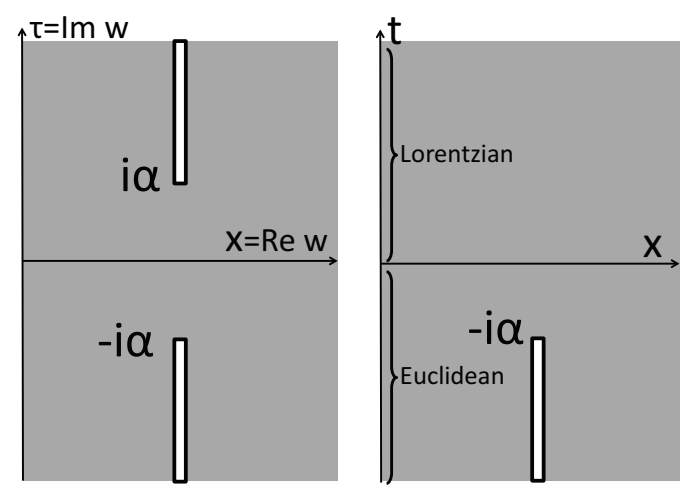

Figure 15. The geometries which realize the joining local quenches. The left figure describes the space on which we perform the Euclidean path-integral. This is mapped into an upper half plane by the map (5.1). The right picture describes the path-integral realization of the time evolution after the joining process happened at $t=0$, where the Euclidean path-integral for $\tau<0$ creates the state just after this process.

\subsection{Joining local quench in Dirac free fermion CFT}

Consider a massless Dirac fermion CFT in this joining quench. The time evolution of EE can be computed from the formula (4.3) and (4.4) as in the previous analysis of splitting quench.

The analytical expressions of $S_{A}$ at the time $t$ when $\alpha$ is infinitesimally small, are given as follows (we choose $A$ to be the interval $a \leq x \leq b$ and we can again assume (4.2) without losing generality). In the early time period, $0<t<|a|$, we have

$$
S_{A}=\frac{1}{6} \log \frac{4|a| b(b-a)^{2}}{(b+|a|)^{2} \epsilon^{2}},
$$

When $|a|<t<b$, we have

$$
S_{A}=\frac{1}{6} \log \frac{4(b-a) b(b-t)\left(t^{2}-a^{2}\right)}{\alpha(a+b)(b+t) \epsilon^{2}},
$$

At late time, $t>b$, we have

$$
S_{A}=\frac{1}{3} \log (b-a) / \epsilon .
$$

It is useful to note that when $b=-a>0, S_{A}$ takes a constant value for any $t: S_{A}=$ $(1 / 3) \log (2 b / \epsilon)=S_{A}^{(0)}$, as we found also for the splitting quench.

The behavior of entanglement entropy is also numerically plotted in figure 16 . The left graph shows that the EE gets larger during only the time period where one of the entangled pair is included in the subsystem A, while the EE is the same as its vacuum value for the other time period. This fact is also true in the above analytical results as well as in all of our numerical plots. This property is consistent with the relativistic particle propagation picture for the massless Dirac fermion CFT. 

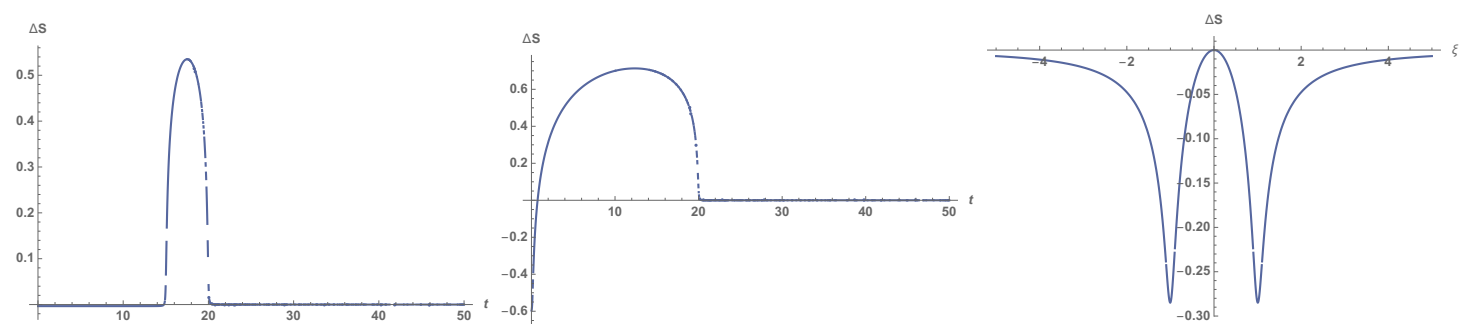

Figure 16. The plots of the entanglement entropy growth $S_{A}-S_{A}^{(0)}$ for $\alpha=0.1$ for the joining local quench in the Dirac Fermion CFT. The left graph describes the time evolution when we take $(a, b)=(15,20)$. The middle one is the time evolution for $(a, b)=(0.1,20)$. The right one is the plot for various $\xi$ when we fix $l=2$ and $t=0$.

The middle graph shows a logarithmic growth at an early time. Indeed, from (5.4), we find that when $|a| \ll t \ll b$, the EE shows the logarithmic behavior:

$$
S_{A} \simeq \frac{1}{3} \log \frac{2 t}{\epsilon}+\frac{1}{6} \log \frac{l}{\alpha}
$$

which agrees with the known local quench behavior [13].

The entanglement density (ED) can also be computed by taking differentiations of this entropy. The results are plotted in figure 17 . We can clearly see the positive peak at $(l, \xi)=(2 t, 0)$ as well as the negative peaks at $(l, \xi)=(0, \pm t)$. The former agrees with the relativistic particle picture. The latter coincides the peaks of the energy density. Even though in the previous splitting case of the Dirac fermion CFT, the negative region $\Delta n<0$ around $(l, \xi)=(t, 0)$ was expanding, in the present joining case, the expanding region at the same place is positive $\Delta n>0$. This indeed violates the naive causality constraint (refer to the right picture in figure 4) and we can again interpret this as the presence of non-local entanglement in the initial state just after the quench. This leads to the logarithmic growth at late time in the joining case (5.6) as opposed to the splitting case.

\subsection{Joining local quench in holographic CFT}

For the holographic CFT, we can substitute the coordinate transformation (5.1) to (2.12) in order to calculate the EE. The dual geometry is given by $\operatorname{Im}[\xi] \geq 0$ in the Poincare AdS coordinate (2.11). The HEE can be computed as (4.10) and (4.11) using the AdS/BCFT, as already analyzed in the earlier paper [17].

The analytical expressions of $S_{A}^{\text {con }}$ and $S_{A}^{\text {dis }}$ at the time $t$ when $\alpha$ is infinitesimally small, are given as follows (we choose $A$ to be the interval $a \leq x \leq b$ with (4.2)). In the early time period, $0<t<|a|$, we have

$$
\begin{aligned}
& S_{A}^{\text {con }}= \begin{cases}\frac{c}{3} \log (b-a) / \epsilon, & (a>0), \\
\frac{c}{6} \log \frac{4\left(a^{2}-t^{2}\right)\left(b^{2}-t^{2}\right)}{\alpha^{2} \epsilon^{2}}, & (a<0)\end{cases} \\
& S_{A}^{\text {dis }}=\frac{c}{6} \log \left(4 b|a| / \epsilon^{2}\right)+2 S_{\mathrm{bdy}} .
\end{aligned}
$$



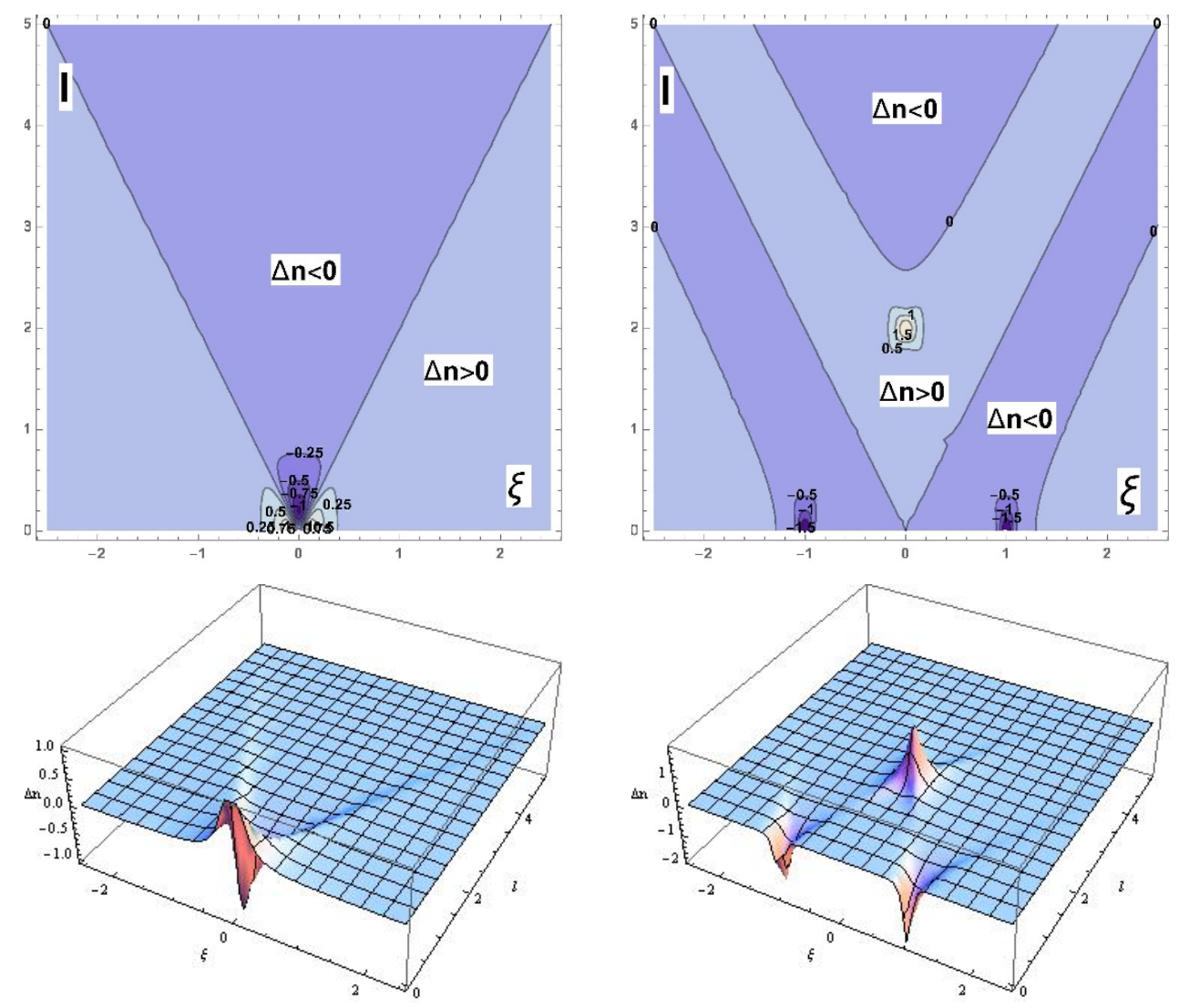

Figure 17. The plots of the entanglement density $\Delta n(\xi, l, t)$ for $\alpha=0.1$ at $t=0$ (left) and $t=1$ (right). The horizontal and depth coordinate corresponds to $\xi$ and $l$, respectively.

When $|a|<t<b$, we have

$$
S_{A}^{\mathrm{con}}=\frac{c}{6} \log \frac{2(b-a)(t-a)(b-t)}{\alpha \epsilon^{2}}, \quad S_{A}^{\mathrm{dis}}=\frac{c}{6} \log \frac{4 b\left(t^{2}-a^{2}\right)}{\alpha \epsilon^{2}}+2 S_{\mathrm{bdy}} .
$$

At late time, $t>b$, we have

$$
S_{A}^{\mathrm{con}}=\frac{c}{3} \log (b-a) / \epsilon, \quad S_{A}^{\mathrm{dis}}=\frac{c}{6} \log \frac{4\left(t^{2}-b^{2}\right)\left(t^{2}-a^{2}\right)}{\alpha^{2} \epsilon^{2}}+2 S_{\mathrm{bdy}} .
$$

In the late time limit $t \gg b$, we always find $S_{A}^{\text {con }}=S_{A}^{(0)}$ dominates as we expect.

The behavior of entanglement entropy is also numerically plotted in figure 18. By comparing the holographic results (figure 18) for vanishing boundary entropy $S_{\mathrm{bdy}}=0$ with those for the Dirac fermion's one (figure 16), we find they agree with each other qualitatively, if we ignore the phase transition behavior in the former. If we take $|a| \ll t \ll$ $b$, then we find the logarithmic growth:

$$
S_{A}=S_{A}^{\mathrm{dis}} \simeq \frac{c}{3} \log \frac{2 t}{\epsilon}+\frac{c}{6} \log \frac{l}{\alpha},
$$

which indeed agrees with the Dirac fermion result (5.6). 


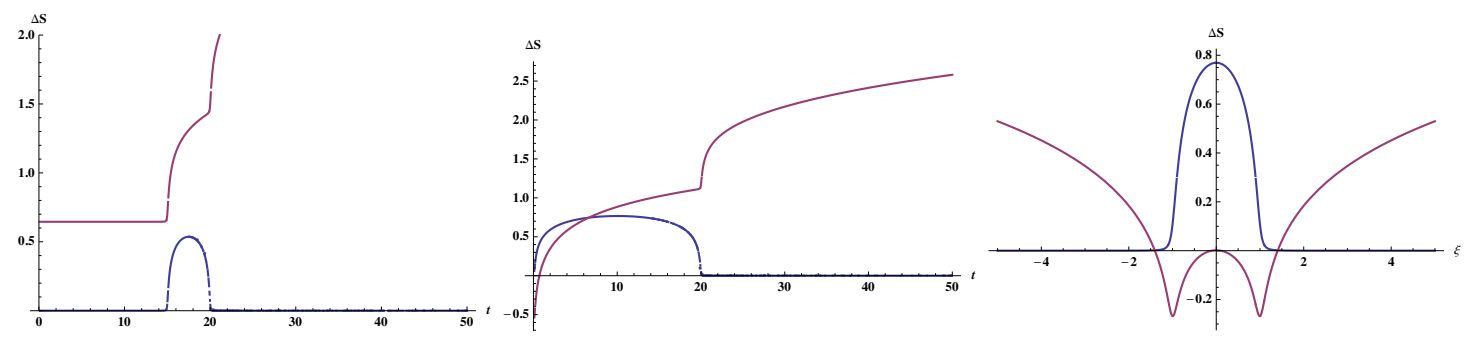

Figure 18. The plots of the holographic entanglement entropy growth $S_{A}-S_{A}^{(0)}$ for $\alpha=0.1$ for the holographic joining local quench. We set the tension vanishing $T_{\mathrm{BCFT}}=0$. The blue and red curve describes $S_{A}^{\text {con }}$ and $S_{A}^{\text {dis }}$, respectively. The left graph describes the time evolution when we take $(a, b)=(15,20)$. The middle one is the time evolution for $(a, b)=(0.1,20)$. The right one is the plot for various $\xi$ when we fix $l=2$ and $t=0$.

However if the boundary entropy is large enough for $S_{A}^{\text {con }}$ to be favored, then we find a slower logarithmic growth for $|a| \ll t \ll b$ :

$$
S_{A}=S_{A}^{\mathrm{con}} \simeq \frac{c}{6} \log \frac{2 t}{\alpha}+\frac{c}{3} \log \frac{l}{\epsilon} .
$$

The entanglement density is plotted in figure 19. In the upper two graphs, we again smeared the derivatives in (3.1) by replacing it with the finite difference. We observe that in addition to the expected positive peak at $(\xi, l)=(0,2 t)$ and the two negative ones at $(\xi, l)=( \pm t, 0)$, we find a continuous peak along the following curve (we again defined $k$ as in $(2.19))$ :

$$
l=t-\frac{|\xi|}{1+k}+\sqrt{\left(t-\frac{|\xi|}{1+k}\right)^{2}+\frac{4 k}{k+1}(t+|\xi|)|\xi|} .
$$

The profile of this curve is plotted in the lower left picture of figure 19. This continuous peak on (4.19) arises since there is a phase transition from $S^{\text {dis }}$ to $S^{\text {con }}$ as we can see from (5.8). At $t=0$, this curve is reduced to the line $l=\frac{2 k}{k+1}|\xi|$. In the limit $t \rightarrow \infty$ and $|\xi| \rightarrow \infty$ we have

$$
l \simeq 2 t+\frac{2(k-1)}{k+1}|\xi|, \quad(t \rightarrow \infty), \quad l \simeq \frac{2 k}{k+1}|\xi|+\frac{4 k}{2 k+1} t, \quad(|\xi| \rightarrow \infty) .
$$

The presence of the codimension one peak is again peculiar to holographic CFTs and is missing in the free Dirac fermion CFTs or more generally RCFTs. Its presence shows that initial entanglement gets modified in highly non-local way. Moreover, this time, the curve (5.12) extends to the region outside of the region $l>2|\xi|$ (i.e. the red region). Therefore the blue curve enters into the red region from both the left and right side as in the lower right picture of figure 19. This explains the logarithmic growth of $S_{A}$ (5.10) with the doubled coefficient compared with that for the local operator quench (3.21). Moreover, if $k$ gets very large, the curve (5.12) approaches to $l \simeq 2|\xi|$ in the limit $|\xi| \rightarrow \infty$. Therefore the situation gets very similar to the local operator quench and therefore this explains the behavior (5.11). Also we will provide the geometric explanation of the $\log t$ behaviors in section 6 . 

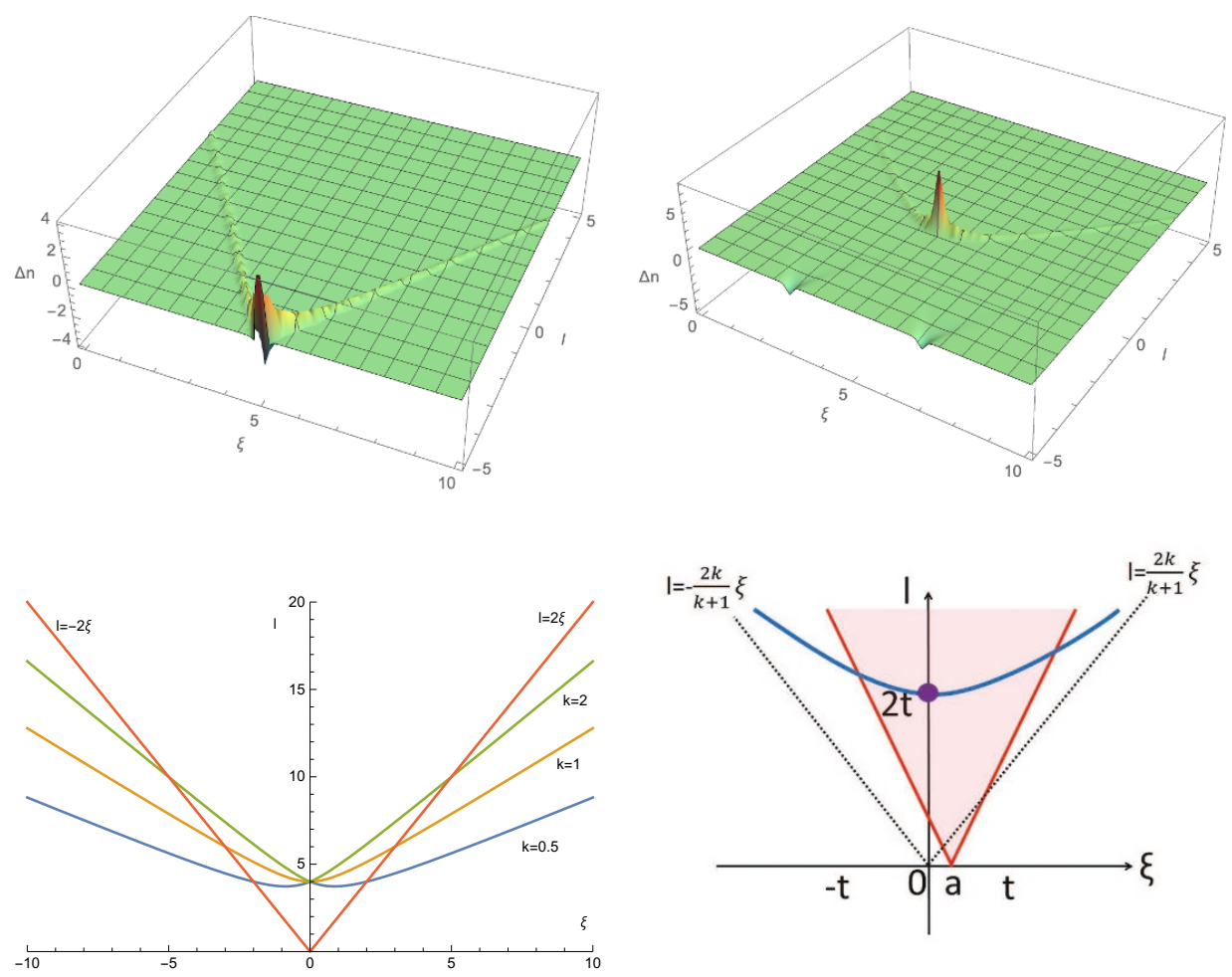

Figure 19. The behaviors of a smeared entanglement density for the holographic joining local quench. The upper left and right graph are the plots of $\Delta n(\xi, l, t)$ at $t=0$ and $t=2$, respectively, with $\alpha=0.1$ (again we set $T_{\mathrm{BCFT}}=0$ ). The horizontal and depth coordinate corresponds to $\xi$ and $l$, respectively. Here we smeared the originally delta functional behavior of the entanglement density by replacing the second derivatives w.r.t. $l$ and $\xi$ with finite-differences $\delta=0.1$. We observe a delta functional behavior on the curve (5.12) at $k=1$. The lower left picture describes the curve (5.12) for $k=0.5$ (blue), $k=1$ (orange) and $k=2$ (green) at $t=2$ as well as the line $l=2|\xi|$ (red). The lower right picture shows the computation of $S_{A}$ for $a \ll t \ll b$ by integrating the ED over the red region. The blue curve represents the peaks on (4.19). As the time evolves, the blue curve enters into the red region from both the left and right side. This explains the logarithmic growth of EE with the doubled coefficient.

\subsection{Holographic geometry of joining local quench}

Now let us study the geometry of the gravity dual for the joining local quench. Since this has many similarities with that for the splitting local quench, our explanation will be brief. Indeed since the energy stress tensor (4.20) remains the same, the metric of gravity dual for the joining quench is the same as that for the splitting one. The difference comes from the location of the boundary surface $Q$. The time slice $t=0$ in the $(w, \bar{w}, z)$ coordinate is again mapped into the quarter sphere $|\xi|^{2}+\eta^{2}=1$ in the half Poincaré AdS: $\operatorname{Im}[\xi]>0$. However the details of the mapping is different from the previous one as sketched in figure 20. In the present case, the region (4.22) corresponds to $\operatorname{Im}[\xi]<0$ and thus should be removed. Its boundary (the red curve) given by $z=2\left(x^{2}+\alpha^{2}\right) / \alpha$ now represents the boundary surface $Q$. The region $-i \alpha<\tau<i \alpha$ and $x=0$ is now mapped into a region $\operatorname{Re}[\xi]=0$ in the half Poincaré $\mathrm{AdS}_{3}$. Since this map is identical to that for the splitting quench i.e. figure 12, we will omit its detail. 


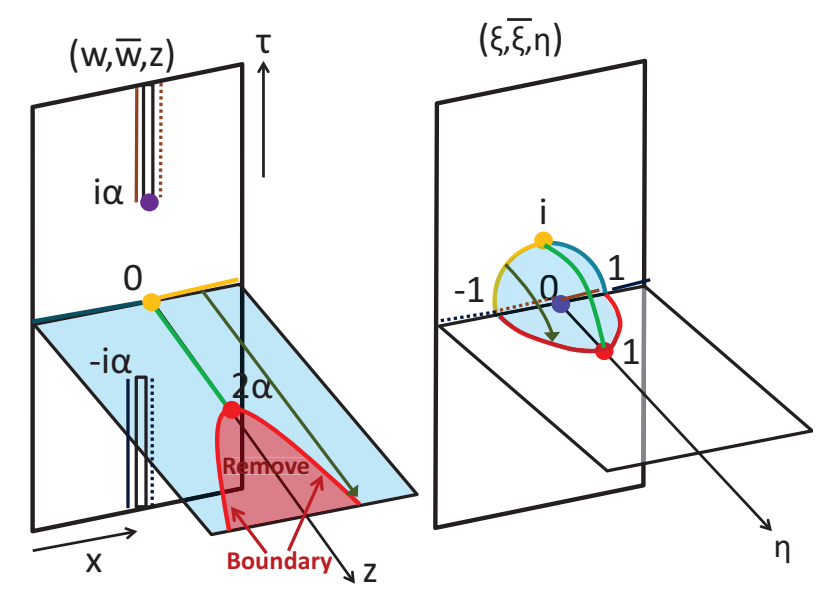

Figure 20. The map (2.12) between the gravity dual of joining local quench (left) and the upper half of the Poincare $\mathrm{AdS}_{3}$ (right). The red curve in the left is given by $z=2\left(x^{2}+\alpha^{2}\right) / \alpha$ and this corresponds to the boundary surface $Q$. Thus the region inside this curve should be removed.

Finally we obtain the spacetime geometry sketched in figure 21 . The boundary surface $Q$, expressed by the red surface, is given by the same expression (4.25) and (4.26) in the Euclidean and Lorentzian case, respectively. In the Euclidean geometry, there is the green surface $Q^{\prime}$ which extends from the CFT boundary and this is actually identified with the red surface $Q$, whose details we will omit as they are involved with complicated numerics. In some sense, in this geometry, the location of the boundary surface $Q$ is opposite to that for the splitting local quenches.

In summary, the time evolution after the joining local quench is described by the spacetime with the boundary surface $Q$ which moves toward the horizon at the speed of light. ${ }^{6}$ This intuitively agrees with our expectation that after the joining, the CFT state gradually approaches to the vacuum state on a connected line. It is also intriguing to note that in the Lorentzian description, the boundary surface $Q$ does not end on the AdS boundary but is localized in the bulk.

So far we implicitly assumed $T_{\mathrm{BCFT}}=0$. For more general cases $T_{\mathrm{BCFT}} \neq 0$, the AdS/BCFT setup in $(\xi, \bar{\xi}, \eta)$ coordinate is given by the boundary surface (4.28). Then we can map $Q$ into the original coordinate $(w, \bar{w}, z)$ as in figure 20 . When $T_{\mathrm{BCFT}}>0$ $\left(T_{\mathrm{BCFT}}<0\right)$, the boundary surfaces $Q$ is moved in the larger (smaller) $z$ direction. In other words, the physical region of the gravity dual, surrounded by the boundary surface $Q$ and the AdS boundary $z=0$, expands as $T_{\mathrm{BCFT}}$ gets larger as expected.

In this subsection we studied the Lorentzian motion of the boundary surface $Q$. For the holographic entanglement entropy, we actually need the profile of geodesic in Lorentzian geometry, though in the previous subsection we employed the formal Wick rotation arguments to avoid this analysis. We will explicitly study how the Lorentzian geodesic looks like in section 6 .

\footnotetext{
${ }^{6}$ This motion of boundary surface is analogous to a string world-sheet description of holographic local quenches proposed in [81], though the choice of geodesics responsible for the HEE is different from ours.
} 


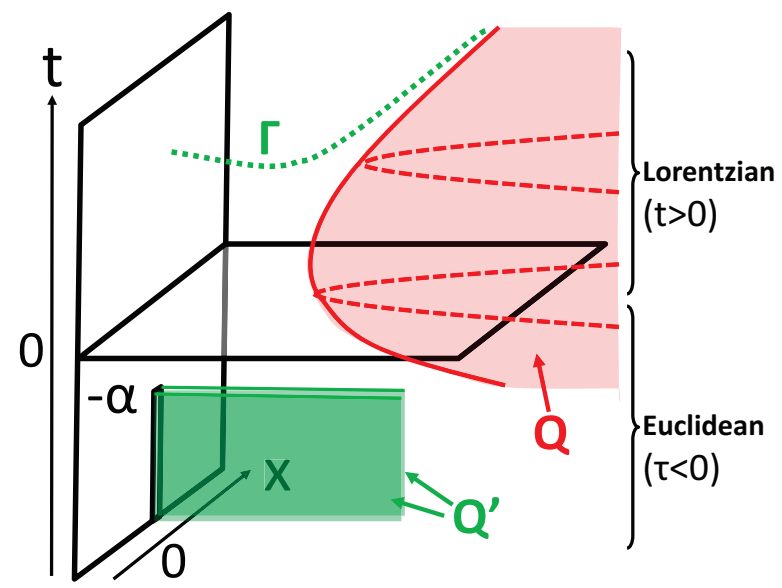

Figure 21. The spacetime geometry of gravity dual of the joining local quench. The red region defined by $z>G_{L}(x, t)$ should be removed. The boundary of this region, which we call the red surface, corresponds to the boundary surface $Q$. Though the green surface $Q^{\prime}$ is also boundary surface, it should be identified with the red one.

\section{Log $t$ geodesic length in holographic Lorentzian geometry}

In our previous calculations of holographic local quenches we mainly worked in Euclidean setups and we took the analytic continuation to Lorentzian time evolutions only for the final results of entanglement entropy. This procedure went straightforwardly or mechanically due to the simplicity of Euclidean gravity duals, while this raises the question whether we really have sensible Lorentzian gravity duals whose geodesic lengths give the correct holographic entanglement entropy. We will confirm this by explicitly finding out the Lorentzian geodesics in this section. Note that in the true Lorentzian gravity dual, coordinates $(t, x, z)$ should take real values, while the coordinates in Poincare coordinate $(\xi, \bar{\xi}, \eta)$ take complex values in general due to the coordinate transformation (2.12) gets complex valued under the Wick rotation.

The results of the time evolutions of HEE under (i) local operator quench $S_{A}^{(O)}$ (3.21), (ii) splitting local quench $S_{A}^{(S)}$ (4.13)-(4.15), and (iii) joining local quench $S_{A}^{(J)}(5.7)-(5.9)$ are characterized by two logarithmic behavior $\log t / \epsilon$ and $\log t / \alpha$. Moreover, we can find the same expression of $S_{A}$ for these different types of local quenches. Our analysis of Lorentzian geodesics in this section, will also explain such logarithmic behaviors in a systematical way.

\subsection{Late time behavior}

First note that in the late time limit $t_{0} \gg b>a$, we find

$$
S_{A}^{\operatorname{con}(S)}\left(t_{0}\right) \simeq S_{A}^{\operatorname{dis}(J)}\left(t_{0}\right) \simeq \frac{c}{3} \log \frac{t_{0}}{\epsilon}+\frac{c}{3} \log \frac{t_{0}}{\alpha} .
$$

First we would like to point out that both are identified with the twice of the geodesic length between the boundary point $(t, x, z)=(t, 0,0)$ and the tip of the surface $Q$, given by

$$
z=\frac{2\left(\alpha^{2}+t^{2}\right)}{\sqrt{\alpha^{2}+4 t^{2}}} \equiv g(t) .
$$


When $t \gg \alpha$, we have

$$
g(t) \simeq t+\frac{7 \alpha^{2}}{8 t}+\cdots
$$

These geodesics are sketch as $\Gamma_{ \pm}$in figure 13 and $\Gamma$ in figure 21 . In the former (splitting quench) case, we consider a connected geodesic and therefore should avoid touching on the boundary surface $Q$. On the other hand, in the latter (joining) case, the disconnected geodesic should end on the boundary surface $Q$. Therefore these two should approximately coincide in the late time limit, by setting $a, b \rightarrow 0$.

Next we would like to explain why the geodesic length looks like (6.1). Instead of finding a geodesic directly in $(t, x, z)$ coordinate, it is easier to start with the transformed coordinate $(\operatorname{Re}[\xi], \operatorname{Im}[\xi], \eta)$. However, we have to note that since we are considering an analytical continuation into the Lorentzian geometry, the coordinates $(\operatorname{Re}[\xi], \operatorname{Im}[\xi], \eta)$ take complex values in general, though $(t, x, z)$ should take real values. By remembering the conformal map (5.1) for the joining quench and the holographic transformation (2.12), it is straightforward to find that the correct geodesic is given by ${ }^{7}$

$$
\xi \bar{\xi}+\eta^{2}=e^{-i \beta}
$$

where the real parameter $\beta(>0)$ is related to the boundary time $t_{0}$ at $z=0$ via

$$
t_{0}=\alpha \cdot \tan \frac{\beta}{2} .
$$

Therefore for $t_{0} \gg \alpha$ we have

$$
\beta \simeq \pi-\frac{2 \alpha}{t_{0}}
$$

In terms of the original coordinate, (6.4) is equivalent to

$$
z=\frac{2\left(t^{2}+1\right)\left(((1+\cos \beta) t-\alpha \sin \beta)^{2}+(t \sin \beta-\alpha(1-\cos \beta))^{2}\right)^{1 / 4}}{\left(\left((1+\cos \beta)\left(3 t+4 t^{3}\right)+\alpha \sin \beta\right)^{2}+\left(\sin \beta\left(3 t+4 t^{3}\right)+\alpha(1-\cos \beta)\right)^{2}\right)^{1 / 4}}
$$

which is plotted in figure 22 .

At late time, we have $z(t) \simeq t-\frac{t_{0}}{2}+O(1 / t)$ and thus the geodesic extends to infinity almost in a light-like way. In the limit $t \rightarrow t_{0} \gg \alpha$ we have

$$
z(t) \simeq \sqrt{t_{0}\left(t-t_{0}\right)}+\frac{\left(t-t_{0}\right)^{3 / 2}}{2 \sqrt{t_{0}}}+\cdots
$$

Indeed we can confirm that this is a solution to the geodesic equation in the holographic spacetime, obtained from (4.20) and (2.13), given by (on $x=0$ ):

$$
\begin{aligned}
d s^{2} & =\frac{1}{z^{2}}\left[d z^{2}-\left(1+f(t, z) z^{2}\right) d t^{2}\right], \\
f(t, z) & =\frac{9 \alpha^{4} z^{2}-24 \alpha^{2}\left(t^{2}+\alpha^{2}\right)^{2}}{16\left(t^{2}+\alpha^{2}\right)^{4}} .
\end{aligned}
$$

\footnotetext{
${ }^{7}$ We can find this form by noting that the solution to the geodesic equation takes the form $\xi \bar{\xi}+\eta^{2}=$ const. and by plugging the coordinate values at the AdS boundary.
} 
We can estimate the geodesic length of (6.7) as

$$
\int_{t_{0}}^{\infty} d t \frac{\sqrt{\left(z^{\prime}\right)^{2}-1-f z^{2}}}{z(t)} \simeq \frac{c}{6} \log \frac{t}{\epsilon}
$$

The twice of this length can explain only one of the logarithmic term in (6.1).

The missing contribution actually comes from another part of the geodesic which is not covered by the coordinate (6.9). Indeed, if we parameterize the geodesic (6.4) as

$$
\xi=e^{-i \beta / 2} \cos \theta, \quad \eta=e^{-i \beta / 2} \sin \theta, \quad\left(0 \leq \theta<\frac{\pi}{2}\right)
$$

Note that $\theta=0$ corresponds to the AdS boundary $\eta=0$ and $\theta=\pi / 2$ corresponds to the boundary surface $Q$ on $\operatorname{Im}[\xi]=0$. Actually from the map (2.12), we find that the trajectory (6.7) for $t_{0} \leq t \leq \infty$ only covers a part of the full geodesic (6.12) given by $0 \leq \theta \leq \beta / 2$. The geodesic length for this part is computed as

$$
\int_{\hat{\epsilon}}^{\cos (\beta / 2) e^{-i \beta / 2}} \frac{e^{-i \beta / 2} d \eta}{\eta \sqrt{e^{-i \beta}-\eta^{2}}}=\log \left(\frac{2 e^{-i \beta / 2}}{\hat{\epsilon}}\right)+\log \left(\frac{\cos (\beta / 2)}{1+\sin (\beta / 2)}\right),
$$

where $\eta=\hat{\epsilon}$ is the cut off corresponding to the original one $z=\epsilon$. The first term in the right hand side is the full contribution

$$
\int_{\hat{\epsilon}}^{e^{-i \beta / 2}} \frac{e^{-i \beta / 2} d \eta}{\eta \sqrt{e^{-i \beta}-\eta^{2}}}=\log \left(\frac{2 e^{-i \beta / 2}}{\hat{\epsilon}}\right) \simeq \log \frac{t_{0}}{\epsilon}+\log \frac{t_{0}}{\alpha},
$$

which agrees with a half of (6.1). The second term is estimated when $t_{0} \gg \alpha$ as

$$
\log \left(\frac{\cos (\beta / 2)}{1+\sin (\beta / 2)}\right) \simeq-\log \frac{t_{0}}{\alpha}
$$

This shows that the part of geodesic length in the Poincaré-like coordinate patch (6.9) gives (identical to $(6.11)$ )

$$
L_{\text {poincaré }}=\log \frac{t_{0}}{\epsilon},
$$

and the one outside of the Poincaré patch does

$$
L_{\text {outside }}=\log \frac{t_{0}}{\alpha} .
$$

To understand this outside contribution well, ${ }^{8}$ let us ignore the back reaction and focus on the Poincaré AdS (restricted on $x=0$ ) by setting $f(t, z)=0$ in (6.9). This is embedded in the global $\mathrm{AdS}_{3}$ (refer to figure 22)

$$
d s^{2}=-\left(r^{2}+1\right) d T^{2}+\frac{d r^{2}}{r^{2}+1}+r^{2} d \phi^{2},
$$

\footnotetext{
${ }^{8}$ Such a contribution is also familiar in holographic global quenches [54-56] and local operator quenches [74].
} 

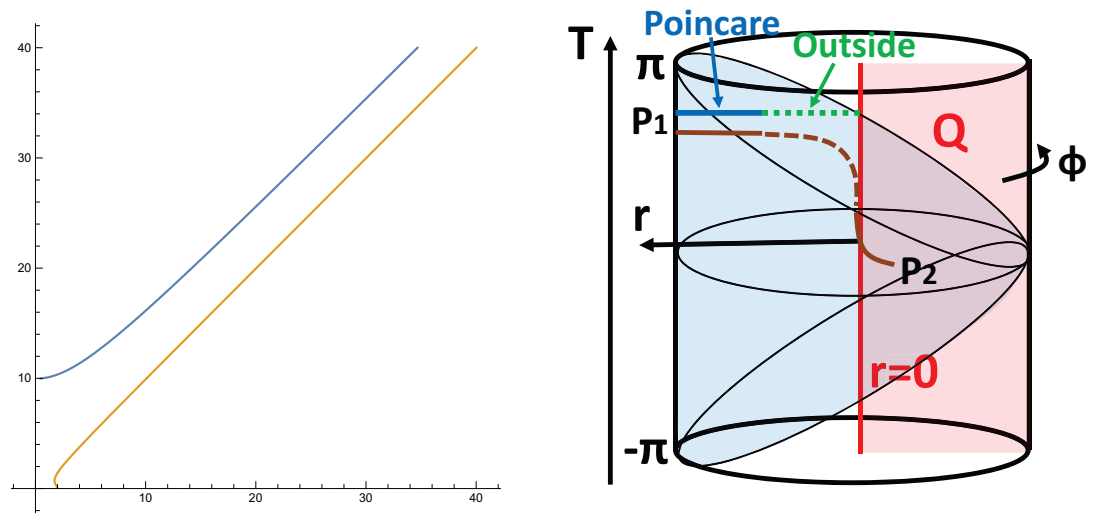

Figure 22. Profiles of the geodesic. In the left graph, we show the geodesic (blue) and the boundary surface $Q$ (orange) in the Poincaré coordinate $(z, t)$ with $t_{0}=10$ and $\alpha=1$. The right picture

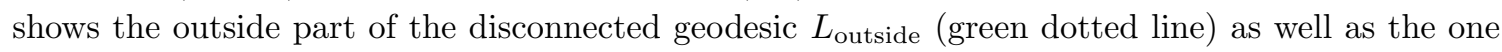
in the Poincaré patch $L_{\text {poincaré }}$ (blue line) in the global AdS coordinate. The point $P_{1}$ is situated at $0<x \ll t$, while the point $P_{2}$ is at $0<t \ll x$. The blue region corresponds to the Poincaré patch. The red vertical line is the tip (6.2) of the boundary surface $Q$. The brown curve describes the connected geodesic that corresponds to (6.21).

at $\theta=0$, via the map (refer to e.g. [11])

$$
\begin{aligned}
& \sqrt{r^{2}+1} \cos T=\frac{\alpha+\left(z^{2}-t^{2}\right) / \alpha}{2 z}, \\
& \sqrt{r^{2}+1} \sin T=\frac{t}{z} .
\end{aligned}
$$

Here the parameter $\alpha$ is introduced such that the static trajectory at $r=0$ is mapped into that for an accelerated particle $z=\sqrt{t^{2}+\alpha^{2}}$ in the Poincare AdS, which imitates the behavior (6.3).

At the AdS boundary, the late time limit $t_{0} \gg \alpha$ in the Poincare AdS corresponds to the time $T \simeq \pi-\alpha / t_{0}$ and the cut off $r_{\infty}=t_{0}^{2} /(\alpha \epsilon)$ in the global AdS. We are considering a geodesic which starts from this boundary point and ends on the tip of the surface $Q$, namely the straight line $r=0$ in the global $\mathrm{AdS}_{3}$. It is clear that this geodesic is given by $T=$ constant and the radial coordinate $r$ runs from $r=r_{\infty}$ to $r=0$. Along this geodesic, the part outside of Poincaré patch is $0<r<r_{*}$, where $r_{*}$ is the solution to $\sqrt{r^{2}+1} \cos T+r=0$, from which we find $r_{*} \simeq t_{0} / \alpha \gg 1$.

Thus, in this simplified model, the geodesic length in the Poincaré patch and that for the outside read (refer to figure 22):

$$
\begin{aligned}
& L_{\text {poincaré }}=\int_{r_{*}}^{r_{\infty}} \frac{d r}{\sqrt{r^{2}+1}} \simeq \log \frac{t_{0}}{\epsilon}, \\
& L_{\text {outside }}=\int_{0}^{r_{*}} \frac{d r}{\sqrt{r^{2}+1}} \simeq \log \frac{t_{0}}{\alpha} .
\end{aligned}
$$

These reproduce the previous results from the exact geodesic (6.16) and (6.17). 


\subsection{Middle time period}

Now we move on to the middle time period and we focus on the region $a \ll t \ll b$ to see the logarithmic behavior clearly. Then we find the following coincidences between the three different types of local quenches: ${ }^{9}$

$$
S_{A}^{\operatorname{con}(O)} \simeq S_{A}^{\operatorname{con}(S)} \simeq S_{A}^{\operatorname{con}(J)} \simeq \frac{c}{6} \log \frac{t_{0}}{\alpha}+\frac{c}{3} \log \frac{b}{\epsilon} .
$$

On the other hand, the disconnected geodesic length in joining quench behaves differently:

$$
S_{A}^{\operatorname{dis}(J)} \simeq \frac{c}{6} \log \frac{t_{0}}{\epsilon}+\frac{c}{6} \log \frac{t_{0}}{\alpha}+\frac{c}{6} \log \frac{b}{\epsilon} .
$$

The coincidence $(6.21)$ can be easily understood because they are described by a geodesic which connects the two end points, where the existence of slits is not a crucial obstruction, and because the background metric is the same (refer to the brown curve in figure 22). We can regard the first term $(c / 6) \log (t / \alpha)$ as the geodesic length outside of the Poincaré patch as in (6.17). The second term $(c / 3) \log (b / \epsilon)$ is simply interpreted as the familiar geodesic length in the Poincare $\mathrm{AdS}_{3}$.

On the other hand, the geodesic length (6.22) is divided into two contributions: the one which connects $x=a$ and $Q$ and the other one which connects $x=b$ and $Q$. The former one is clearly equal to $(c / 6)\left(L_{\text {poincare }}+L_{\text {outside }}\right)$ in (6.16) and (6.17). The latter one is simply given by the standard result of AdS/BCFT: $(c / 6) \log (b / \epsilon)$. These explain the behavior (6.22).

\section{Tensor network interpretation of local quenches}

Here we would like to present a sketch of tensor network description of time evolution of our local quench states and their entanglement entropies so that they matches with our gravity duals. Our argument in this section will be heuristic and qualitative.

We employ the MERA (Multiscale Entanglement Renormalization Ansatz) [20, 21] for our qualitative interpretation, though our arguments can equally hold for other holographic tensor networks. MERA is a class of tensor networks which are constructed by two kinds of tensors: isometry and disentangler (figure 23). This is manifestly scale invariant and is known to describe ground states of critical quantum systems with high accuracy. In tensor networks, by contracting all interior tensors, a desired quantum state is realized at the boundary. We regard the physical system as the boundary, and the tensor network structure as the bulk in AdS/CFT [19].

Consider a curve $\gamma$ in the bulk, whose length can be defined by the number of tensors it crosses. ${ }^{10}$ For a subsystem $A$ on the boundary, its EE $S_{A}$ is upper bounded by

$$
S_{A} \leq C \times\left(\text { length of } \gamma_{A}\right)
$$

\footnotetext{
${ }^{9}$ Note that for the holographic local operator quench, we need only connected geodesics as there is no boundary surface $Q$.

${ }^{10}$ It would be more precise to define the length of a curve in a tensor network by the number of tensor legs it crosses. In our case, however, since we only care about the shortest curve in MERA, this definition is also allow for convenience.
} 

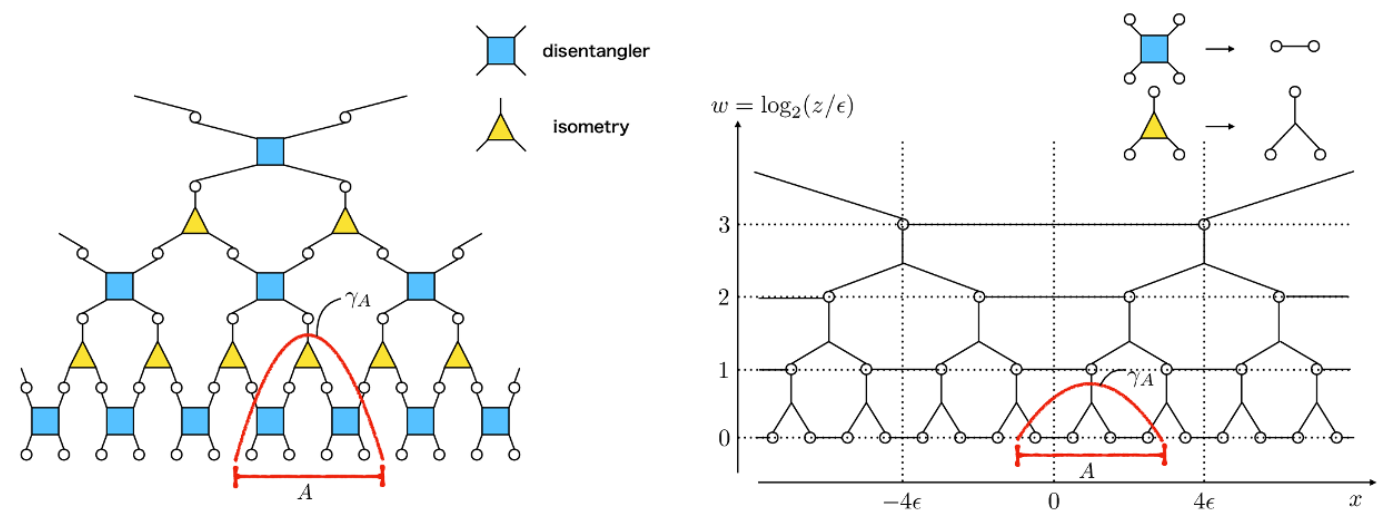

Figure 23. (Left) A standard description of MERA tensor network. (Right) A simplified representation of MERA. This representation can help us capture the relationship between MERA and AdS. (Both) $A$ is a subsystem of the boundary of MERA, and $\gamma_{A}$ is a curve in the bulk who share the boundary with $A$. $(z, x)$ forms a Poincaré coordinate, while $w=\log _{2}(z / \epsilon)$ corresponds to the number of layer.

where $C$ is a constant and $\gamma_{A}$ is an arbitrary curve who shares the boundary with $A$ (i.e. $\partial \gamma_{A}=\partial A$ ).

Particularly, in typical examples of MERA, such as that of a critical transverse Ising model, $S_{A}$ is roughly equal to

$$
S_{A} \simeq C \times\left(\text { length of } \gamma_{A}^{\min }\right)
$$

where $\gamma_{A}^{\min }$ is the minimal curve, whose length is proportional to $\log L_{A}$, with $L_{A}$ the size (i.e. the number of spins) of subsystem $A$.

A qualitative correspondence between MERA and AdS is as follows [19]. If we set the coordinate $x$ parallel to the layers, and the coordinate $w=\log _{2}(z / \epsilon)$ vertical to them (the right picture in figure 23), then the geometry of MERA is interpreted as a discrete version of the time slice of AdS (i.e. hyperbolic space), whose metric is

$$
d s^{2}=\left(\frac{d z^{2}+d x^{2}}{z^{2}}\right)=R^{2} \epsilon^{-2} \frac{d w^{2}}{\left(\log _{2} e\right)^{2}}+2^{-2 w} d x^{2},
$$

where $\epsilon$ is introduced as an UV cutoff. Refer to [34] for a possible interpretations of various slices in AdS spacetime as tensor networks.

Now we would like to consider an tensor network interpretation of our three types of local quenches (local operator/splitting/joining). We will take a heuristic strategy: first we assume specific structures of tensor networks under the time evolutions and then confirm if they reproduce the correct time evolutions of entanglement entropy. In our construction of tensor networks corresponding to local quenches, we assume that an appropriate time slice $t=$ const. of a gravity dual spacetime after a local quench, is approximately a tensor network at the time $t$. We note that the information of tensor network at each time can be separated in to two ingredients: one is the geometric structure of tensor network itself, 


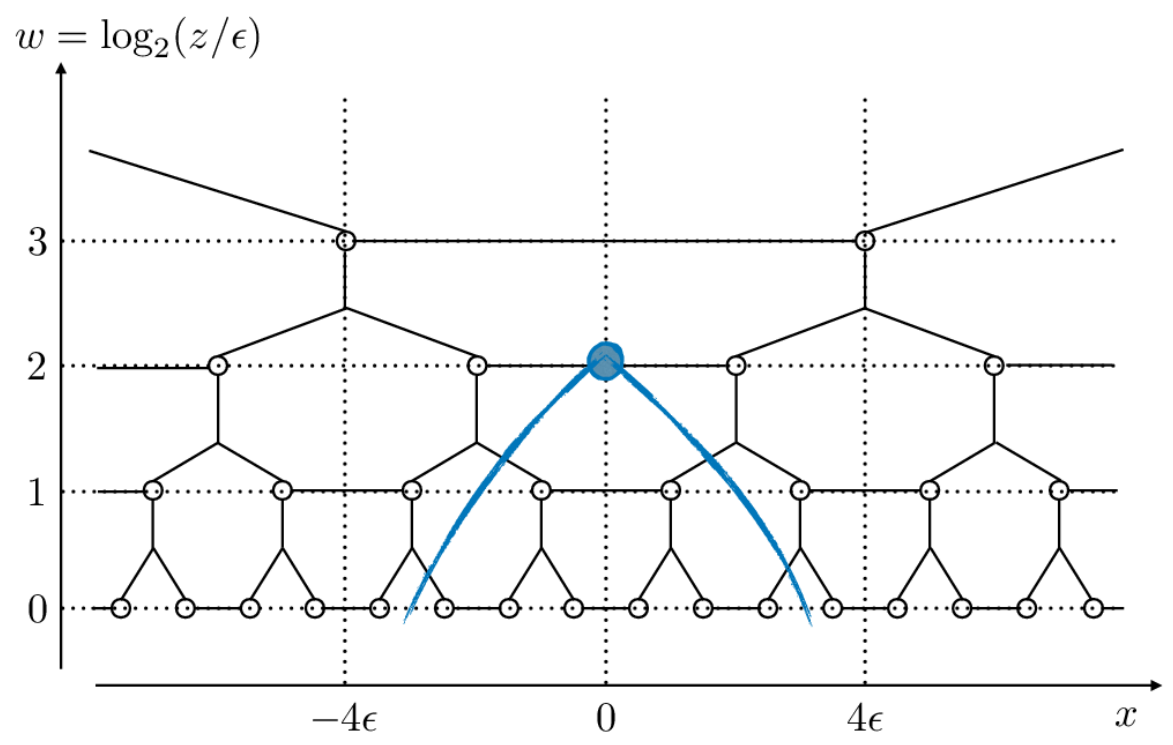

Figure 24. A tensor network description of local operator quench at $t=4 \epsilon$. The blue dot is the large modification of tensor dual to the falling particle. The blue curves describe the image of shock wave deformation of tensors. The parameters of tensors are expected to be modified, and tensors close to the blue curve are expected to accept the strongest modification.

and another one is the parameters of each tensor. ${ }^{11}$ One essential observation we will make below is that we can divide the growth of EE into a "shock wave contribution" and "splitting/joining contribution", which can also be suggested from the holographic analysis in the previous section. In the discussion below, we will regard the "shock wave contribution" as modifications of the parameters and "splitting/joining contribution" as modifications of the network structure. We will see that under this assumption, by choosing appropriate tensor network structure and neglecting modifications on the parameters, we can realize qualitative features of the entanglement structure in the AdS/CFT setup.

\subsection{Local operator quench}

A local operator quench in a holographic CFT can be regarded as a particle falling towards the AdS black hole horizon on the gravity side [14]. This particle trajectory is analogous to the tip of the boundary surface $Q$ in the splitting/joining local quench. In this picture, its back-reaction on spacetime spreads as a shock wave [82], and the EE of a subregion on the boundary will change when the shock wave crosses its minimal surface.

Accordingly in the tensor network, we expect the local operator quench causes a modification of tensor at $x=0$ and $w=0$ due to the initial operator insertion, though the structure of tensor network does not change. As the time evolves, this modification propagates into the interior as $w \sim \log t$. At the same time, tensors are modified along the shock

\footnotetext{
${ }^{11}$ In a MERA corresponding to the ground state of a critical system, for example, network structure means the way tensors linked with each other. Besides, all the isometries (disentanglers) carry the same parameters due to the scale invariance of the state. Network structure and parameters together gives a corresponding quantum state.
} 
wave. These modifications increases quantum entanglement locally. These are depicted in figure 24.

The behavior of the EE is consistent with the causal behavior that the EE gets nontrivial only for the time interval $a<t<b$, as we found both for the RCFT and holographic CFT. In the holographic case (3.21), however, we observed a logarithmic growth

$$
S_{A} \simeq \underbrace{\frac{c}{6} \log \frac{t}{\alpha}}_{\text {shock wave }}+\ldots,
$$

if $a \ll t \ll b$. Our entanglement density analysis showed such a logarithmic contribution is due to the presence of non-local entanglement at the initial state. Our geometric analysis in the gravity dual shows this contribution comes from outside of the Poincaré patch (6.17). In tensor network, this hidden contribution is expected to come from modifications of tensor parameters, though we cannot figure it out precisely. Since this is owing to the huge back-reactions, we would like to still call this a "shock wave contribution". To find out the details of this contribution, we need to make the conjectured correspondence between the AdS/CFT and tensor networks more precisely beyond qualitative arguments, which is not available at present.

\subsection{Splitting quench}

Now we moved on to the splitting quench. We split the disentangler in the MERA at $x=0, z=\epsilon(w=0)$, initially (refer to [83] for introducing boundaries in MERA). This is motivated by the gravity dual where the boundary surface extends to the bulk as in figure13. We identify the boundary in the gravity dual as the termination of tensor network (i.e. cutting the disentanglers or unitaries). This elimination of disentanglers propagates under the time evolutions as in the gravity dual. Therefore at the time $t=2^{n} \epsilon$, the disentangler at $x=0, w=n$ is eliminated. At the same time, from the gravity dual, we expect there are shock wave propagations as in the local operator quench case. Thus we also expect that in our tensor network description a shock wave spreads from $w=n$ at the same time $t=2^{n} \epsilon$. Both of them will contribute to the change of EE. We call them "shock wave contribution" and "splitting contribution" respectively.

From the CFT viewpoint, we can heuristically understand this prescription as follows. When we triggered the splitting quench, we eliminate the nearest neighbor interactions between two adjacent lattice sites. This is equivalent to cutting the disentangler in the very UV. After the time evolution this cutting of entanglement propagates towards the IR.

We show in figure 25 how the network changes after splitting quench. If we focus on the subsystem $A=\{x \mid x \in(0, l \epsilon)\}$ and neglect modifications of tensor parameters (equally neglect "shock wave contribution"), we will find $S_{A}$ is given by the length of minimal disconnected curve, which decreases logarithmically in time at first, and then stays as a constant. In other words, splitting contribution gives a negative logarithmic growth $\propto-\log t / \epsilon$ to $S_{A}^{\text {dis }}$. On the other hand, as we have mentioned in the case of local operator quench (7.4), the shock wave contribution should give a positive logarithmic 


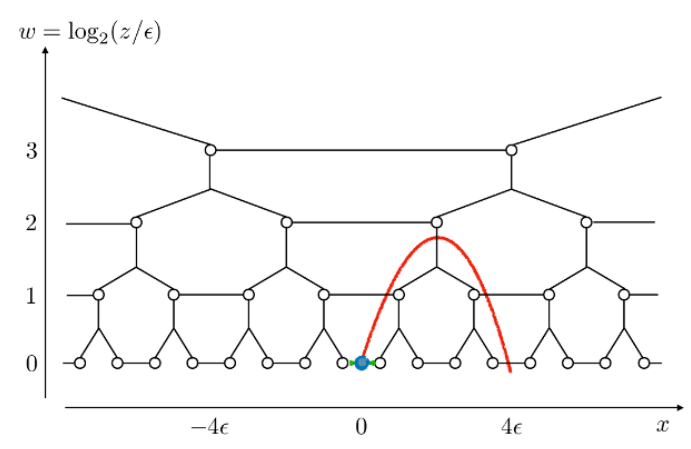

$t=\epsilon$

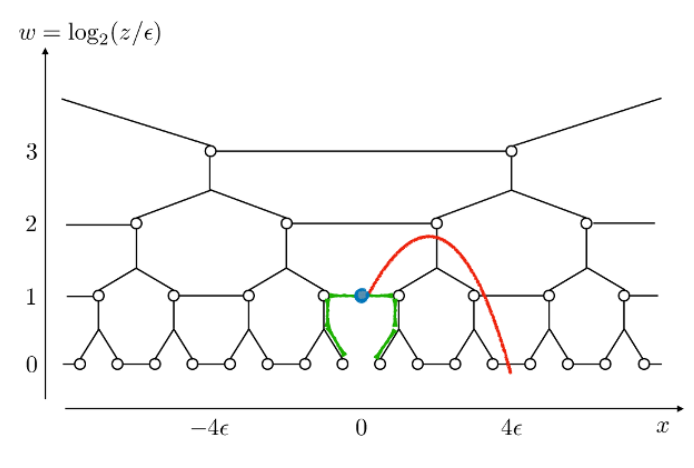

$t=2 \epsilon$

Figure 25. Time evolution of the tensor network after splitting quench. The red curve shows the minimum curve in the bulk. The blue dot and green curve are dual to the falling particle and boundary surface $Q$. Here, $A=\{x \mid x \in(0,4 \epsilon)\}$.

growth $\propto \log t / \alpha$ to EE. In this way, we reach the following estimation:

$$
S_{A}^{\mathrm{dis}} \simeq \underbrace{\frac{c}{6} \log \frac{t}{\alpha}}_{\text {shock wave }} \underbrace{-\frac{c}{6} \log \frac{t}{\epsilon}}_{\text {splitting }}+\ldots,
$$

where we determined the coefficient of the second logarithmic term so that we do not totally have any logarithmic growth as in our previous calculation of HEE (4.14).

\subsection{Joining quench}

For the joining local quench, we start with two copies of semi infinite MERA, which are disconnected initially. The quench is triggered by adding a new disentangler on $x=0, z=$ $\epsilon(w=0)$, at $t=\epsilon$. Then, besides a shock wave spreading at $w=n$ in the bulk, a new disentangler on $x=0$ comes out to connect the $n$th layer at $t=2^{n} \epsilon$. Both of them will contributes to the EE evolution. We call them "shock wave contribution" and "joining contribution" respectively.

We show in figure 26 how the network will change after joining quench. If we focus on block $A=\{x \mid x \in(0, l \epsilon)\}$ and neglect modifications of tensor parameters (equally neglect "shock wave contribution"), we will find $S_{A}$ is firstly given by the length of minimal disconnected curve, which increases logarithmically in time. In other words, the joining contribution gives a positive logarithmic growth to $S_{A}^{\text {dis }}$, which is expected to be equal to the absolute value of splitting contribution, since they should cancel with each other if they occur at the same time. On the other hand, since the shock wave contribution should give a positive logarithmic growth that cancels with split contribution, it is proper to think that the two contributions together give a double logarithmic growth, which is exactly consistent with the results in section 5.2. That is,

$$
S_{A}^{\text {dis }} \simeq \underbrace{\frac{c}{6} \log \frac{t}{\alpha}}_{\text {shock wave }}+\underbrace{\frac{c}{6} \log \frac{t}{\epsilon}}_{\text {joining }}+\ldots
$$

In this way, we can explain the logarithmic growth we saw in (5.8) 

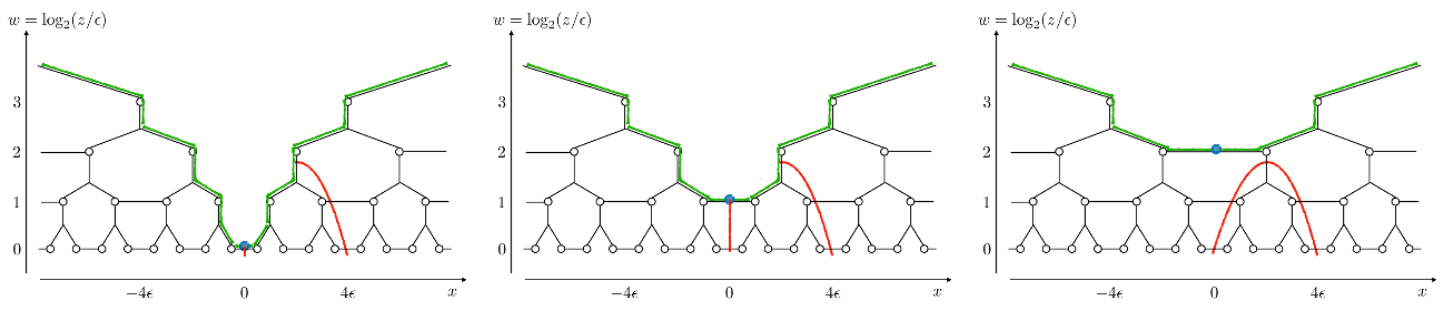

Figure 26. Time evolution of the tensor network after joining quench. The left, middle and right picture correspond to $t=\epsilon, 2 \epsilon$ and $4 \epsilon$, respectively. The red curve shows the minimum curve in the bulk. The blue dot and green curve are dual to the falling particle and boundary surface $Q$. Here, $A=\{x \mid x \in(0,4 \epsilon)\}$.
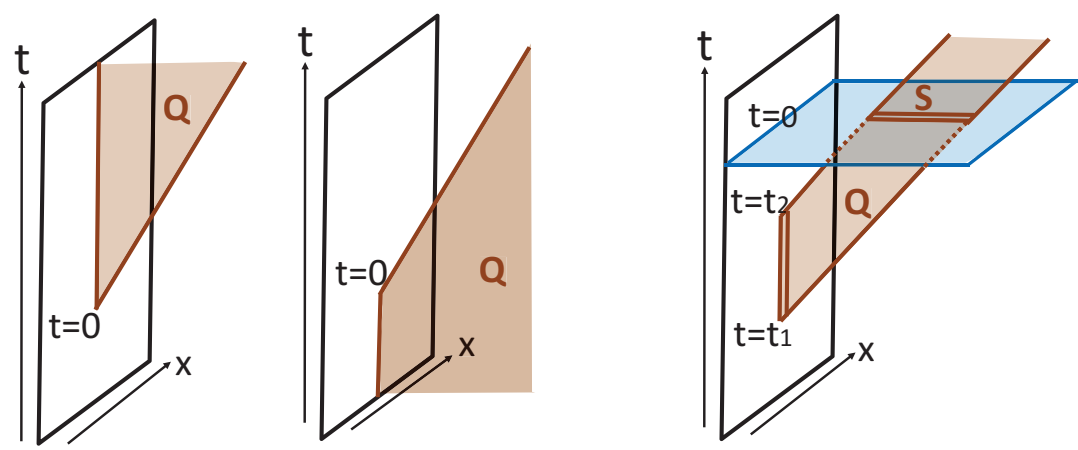

Figure 27. A simplified sketch of the gravity dual spacetimes for the splitting (left), the joining (middle) local quench and their combination (right). The brown surfaces describe the boundary surfaces $Q$. In the right picture, the boundary surface $Q$ intersects with the blue horizontal plane which describes the time slice $t=0$. Their intersection $S$ makes a slit on the time slice.

\section{Holographic quantum circuits from AdS/BCFT}

We can simply summarize gravity dual spacetimes of the splitting and joining local quench as in the left and middle picture of figure 27 in the limit $\alpha \rightarrow 0$. If we combine them such that we join two CFTs at $t=t_{1}$ and split them again at $t=t_{2}$, then the expected holographic dual is given by the right picture in figure 27. The important lesson from this example is that we can create a slit ( $S$ in that picture) which looks "floating" in the bulk on a time slice of gravity dual. Of course, the location of such a floating slit moves under the time evolution following the condition (2.17).

As an ambitious attempt which finalizes this paper, we would like to propose a holographic counterpart of tensor network states in the AdS/BCFT setup by repeating such procedures. ${ }^{12}$ Consider the MERA tensor network [20, 21] and try to realize an analogous geometry on a time slice of a gravity dual. As we explained in section 7 , the MERA network consists of the disentangling and coarse-graining operations, which cut the entanglement in the ground state of a critical spin chain. We depicted a simple setup to achieve this in figure 28. In this analogy, each "spin" in MERA corresponds to the (blue) region

\footnotetext{
${ }^{12}$ For another connection between BCFTs and tensor networks refer to [84].
} 
between two (brown) slits. A coarse-graining and disentangler correspond to terminating and creating a hole in a (brown) slit, respectively. The standard estimation of $\mathrm{EE} S_{A}$ in the MERA nicely agrees with the HEE calculation in the AdS/BCFT as we show in figure 28, where we simply ignored the back-reactions. The entanglement entropy reduced by the coarse-graining/disentangler operation correspond to the green/red dotted line in the pictures.

Notice that in this section we consider a different interpretation of the AdS spacetime in terms of tensor networks, as compared with the arguments in section 7. Here we approximately regard the narrow strip in AdS space as a link in a tensor network and fill the gap between the discretized lattice network (such as MERA) between the continuous AdS spacetime by cutting the latter spacetime into lattices by inserting various boundary surfaces of AdS/BCFT. This idea opens up a new approach to the conjectured connection between the AdS/CFT and tensor networks.

Next we discuss how to realize a gravity dual whose time slice is given by the geometry in figure 28. One way is to perform an Euclidean path-integration on the manifold given by the left picture in figure 28 with $z$ interpreted as the Euclidean time $-\tau$ and consider its gravity dual. Note that the initial state $z=-\tau=\infty$ is expected to be a completely disentangled state. Therefore we choose the state at $\tau=-\infty$ to be the (regularized) boundary state $|B\rangle[85,86]$. Indeed, we expect that the time slice $\tau=0$ of such a Euclidean gravity dual is given by what we want, according to what we learn from the holographic local quenches summarized in figure 27.

Another idea is based on a Lorentzian path-integration in the dual CFT. Now we regard $z$ as the real time $-t$ in figure 28 and again choose the initial state at $t=-\infty$ to be the boundary state $|B\rangle$. Since each end points of the slits moves toward the horizon at the speed of light, we again expect that the gravity dual of this time-dependent setup is approximately given by the one in figure 28 .

\section{Conclusions}

In this paper, we studied the time evolutions of entanglement entropy (EE) under three types of local quenches (local operator, splitting and joining) in two dimensional CFTs. Our main examples of CFTs are the free massless Dirac fermion CFT and holographic CFTs in two dimensions. The typical behavior of EE for each of the three types of local quenches in holographic CFTs is the logarithmic growth for a large subsystem. On the other hand, for operator local quenches in free or RCFTs, we observe simple step functional behaviors. However, for splitting and joining local quenches, we still have logarithmic time evolutions even for free or RCFTs. We also noted that there are two types of logarithmic growth: $(c / 6) \log (t / \alpha)$ and $(c / 6) \log (t / \epsilon)$, where $\alpha$ is a regularization parameter of the local quench and $\epsilon$ is the lattice spacing of the CFT. In this paper, we got systematic understandings of these differences from various points of view, including entanglement density (ED), holographic geometry, and tensor network descriptions. We also calculated evolution of EE in spin systems for splitting quenches. 

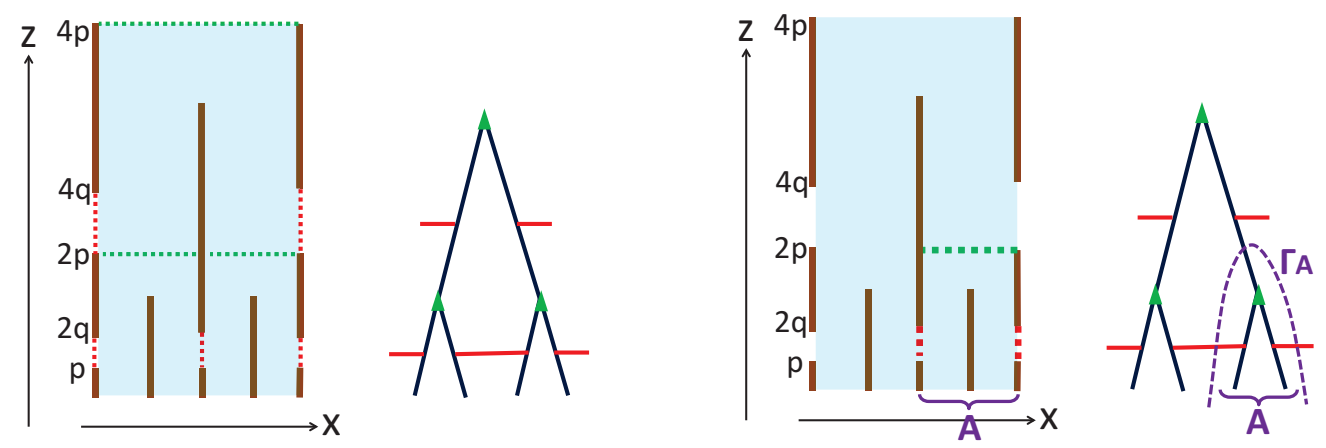

Figure 28. A sketch of the time slice of our proposed $\mathrm{AdS}_{3} / \mathrm{BCFT}_{2}$ setup dual to a MERA-like tensor network (left two pictures), and the estimation of EE $S_{A}$ in the AdS/BCFT and tensor network (right two pictures). The blue region in each picture is the bulk time slice and we added its tensor network description in its next right. The brown thick lines are the slits created by the boundary surface $Q$ in the AdS/BCFT. The red lines and green triangles in the tensor network describe the disentanglers and the coarse-graining tensors. The red and green dotted lines are the geodesics whose length compute the holographic entanglement entropy, corresponding to the entanglement removed by the disentanglers or coarse-graining tensors. We ignore the back-reactions just for simplicity and choose the locations of the slits such that the values of the EE for each red/green dotted lines are the same, which leads to the scale invariance. By comparing the right two pictures, we find that the calculation of HEE in our AdS/BCFT setup is equivalent to that for the tensor network.

In the holographic CFT case, the EE is computed as the length of geodesics in the dual $\mathrm{AdS}_{3}$ spacetime based on the holographic entanglement entropy (HEE). Furthermore, in the splitting/joining quench case, these geodesics can end on the boundary surfaces $Q$, following the AdS/BCFT prescription. Therefore there are two kinds of geodesics: connected and disconnected. Thus the HEE is given by $\min \left\{S_{A}^{\text {con }}, S_{A}^{\text {dis }}\right\}$. One of them, $S_{A}^{\text {dis }}$, depends on the tension parameter $T_{\mathrm{BCFT}}$ in AdS/BCFT.

In the splitting and joining quench, both gravity duals share the same metric but have different boundary surfaces $Q$. In order to see the logarithmic growth of HEE, we have to consider a region which is not covered by the Poincaré patch because the geodesic penetrates the Poincaré horizon. We found that the growth $(c / 6) \log (t / \epsilon)$ comes from the geodesic length in the Poincaré patch, while the other one $(c / 6) \log (t / \alpha)$ comes from that near the surface $Q$, which is hidden inside the Poincaré horizon.

In the splitting quench, the boundary surface $Q$ expands from the AdS boundary toward the bulk at the speed of light. We can regard the two semi-infinite lines at each time are connected through an expanding horizon or equally (non-traversal) wormhole. Note that since $Q$ is time-like, there is no causal influence between them. In the joining quench, the boundary surface $Q$ can be regarded as a "falling string" towards the horizon, which is analogous to the falling particle in the holographic local operator quench. In both examples, the precise location of $Q$ depends on the tension $T_{\mathrm{BCFT}}$ in a way that when $T_{\mathrm{BCFT}}>0$ (or $T_{\mathrm{BCFT}}<0$ ), the region of gravity dual expands (or shrinks).

Entanglement density (ED) turns out to be a good tool to systematically probe entanglement structures between two space points. The analysis with the ED helped us to 
figure out that the initial entanglements created by our "local" quenches is highly nonlocal, except for the local operator quench in RCFTs. In general, the "local" quenches are not described as local unitary transformations. In holographic case, the ED can straightforwardly explain logarithmic time evolutions under different local quenches. However, the two evolutions $(c / 6) \log (t / \alpha)$ and $(c / 6) \log (t / \epsilon)$ cannot be easily distinguished from this perspective.

The gravity duals of local quenches also have a qualitative interpretation in terms of MERA-like tensor networks, where $\sim \log (t / \epsilon)$ behavior can be understood as splitting/joining disentanglers. However, we could not find a clear realization of the $\sim \log (t / \alpha)$ behavior, called "shock wave" contribution. One possibility is to explain this by inserting extra tensors into the networks and we would like to leave more details for a future problem.

In the final part of this paper, we presented an analogue of quantum circuits using both of splitting and joining quenches and gave their gravity duals based on the AdS/BCFT. This offers a method of discretizing gravity dual spacetimes. It is expected that this can further lead to a deeper understanding of the conjectured AdS/tensor network correspondence.

\section{Acknowledgments}

We are grateful to Pawel Caputa, Masahiro Nozaki, Tokiro Numasawa and Tomonori Ugajin for useful comments. TT is supported by the Simons Foundation through the "It from Qubit" collaboration and by World Premier International Research Center Initiative (WPI Initiative) from the Japan Ministry of Education, Culture, Sports, Science and Technology (MEXT). TT is supported by JSPS Grant-in-Aid for Scientific Research (A) No.16H02182 and by JSPS Grant-in-Aid for Challenging Research (Exploratory) 18K18766.

\section{A Vacuum entanglement density in massless Dirac fermion CFT}

Here we would like to show that the simple profile of entanglement density (we set $c=1$ in $(3.3))$

$$
n_{0}(x, y)=\frac{1}{6(x-y)^{2}},
$$

reproduces arbitrary entanglement entropy in the massless Dirac fermion CFT, including cases where the subsystem $A$ consists of multiple disconnected intervals:

$$
A=I_{1} \cup I_{2} \cup \cdots \cup I_{n} .
$$

We choose the $n$ intervals are parameterized by $I_{i}=\left[a_{i}, b_{i}\right]$ for $i=1,2, \cdots, n$. For convenience, we introduce $b_{0}=-\infty$ and $a_{n+1}=\infty$. Then, by summing all bipartite entanglement, using the entanglement density (A.1), $S_{A}$ is estimated as follows:

$$
\begin{aligned}
S_{A} & =\sum_{k=0}^{n} \sum_{l=1}^{n} \int_{b_{k}}^{a_{k+1}} d x \int_{a_{l}}^{b_{l}} d y \frac{1}{6(x-y)^{2}}, \\
& =\frac{1}{6} \sum_{k=0}^{n} \sum_{l=1}^{n}\left(\log \left(\frac{a_{k+1}-b_{l}}{a_{k+1}-a_{l}}\right)+\log \left(\frac{b_{k}-a_{l}}{b_{k}-b_{l}}\right)\right), \\
& =\frac{1}{3} \sum_{i, j=1}^{n} \log \left|b_{i}-a_{j}\right|-\frac{1}{6} \sum_{i, j=1}^{n} \log \left|a_{i}-a_{j}\right|-\frac{1}{6} \sum_{i, j=1}^{n} \log \left|b_{i}-b_{j}\right| .
\end{aligned}
$$


We interpret the diagonal terms in the final expression by introducing the UV cut off $\epsilon$ as $a_{i}-a_{i}=b_{i}-b_{i}=\epsilon$. Thus finally we obtain

$$
S_{A}=\frac{1}{3} \sum_{i, j=1}^{n} \log \frac{\left|b_{i}-a_{j}\right|}{\epsilon}-\frac{1}{3} \sum_{i>j=1}^{n} \log \frac{\left|a_{i}-a_{j}\right|}{\epsilon}-\frac{1}{3} \sum_{i>j=1}^{n} \log \frac{\left|b_{i}-b_{j}\right|}{\epsilon} .
$$

This indeed agrees with the known expression of entanglement entropy in the massless Dirac fermion CFT $[9,10]$. A closely related property is that the multi partite mutual information is vanishing [87].

\section{B Detailed computations of evolutions of holographic entanglement en- tropy}

Here we present the details of calculations of HEE under splitting/joining local quenches. We define the Euclidean coordinate $(\tau, x)$ and complex coordinate $(w, \bar{w})$ as

$$
w=x+i \tau, \quad \bar{w}=x-i \tau .
$$

Both splitting/joining quench setup are described by almost the same conformal maps:

$$
\begin{array}{rll}
\text { (splitting) } & f_{+}(w)=i \sqrt{\zeta(w)} & \arg (\zeta) \in(-\pi, \pi] \\
\text { (joining) } & f_{-}(w)=i \sqrt{-\zeta(w)} & \arg (-\zeta) \in(-\pi, \pi]
\end{array}
$$

Here we define $\zeta(w)$ by

$$
\zeta(w)=\frac{w+i \alpha}{w-i \alpha}
$$

The HEE for the connected/disconnected geodesics $S_{A, \pm}^{\text {con }}, S_{A, \pm}^{\mathrm{dis}}$ for the subsystem $A(=$ $\left.\left[w_{a}, w_{b}\right]\right)$ is computed by (when the tension $T_{\mathrm{BCFT}}$ is vanishing):

$$
\begin{aligned}
S_{A, \pm}^{\text {con }} & =\frac{c}{12} \log \left(\frac{\left|f_{ \pm}\left(w_{a}\right)-f_{ \pm}\left(w_{b}\right)\right|^{4}}{\epsilon^{4}\left|f_{ \pm}^{\prime}\left(w_{a}\right)\right|^{2}\left|f_{ \pm}^{\prime}\left(w_{b}\right)\right|^{2}}\right) \\
S_{A, \pm}^{\text {dis }} & =\frac{c}{12} \log \left(\frac{16\left(\operatorname{Im} f_{ \pm}\left(w_{a}\right)\right)^{2}\left(\operatorname{Im} f_{ \pm}\left(w_{b}\right)\right)^{2}}{\epsilon^{4}\left|f_{ \pm}^{\prime}\left(w_{a}\right)\right|^{2}\left|f_{ \pm}^{\prime}\left(w_{b}\right)\right|^{2}}\right) .
\end{aligned}
$$

The plus and minus subscript in $S_{A, \pm}$ corresponds to splitting and joining quench respectively.

When computing these quantities, it will be helpful to use the results below.

$$
\begin{aligned}
\zeta=\frac{w+i \alpha}{w-i \alpha} & =\frac{x+i(\tau+\alpha)}{x+i(\tau-\alpha)}=\frac{x^{2}+\tau^{2}-\alpha^{2}+i(2 x \alpha)}{x^{2}+(\tau-\alpha)^{2}} \\
|\zeta| & =\frac{\sqrt{\left(x^{2}+\tau^{2}-\alpha^{2}\right)^{2}+(2 x \alpha)^{2}}}{x^{2}+(\tau-\alpha)^{2}}=\sqrt{\frac{x^{2}+(\tau+\alpha)^{2}}{x^{2}+(\tau-\alpha)^{2}}} \\
\frac{d \sqrt{ \pm \zeta}}{d w} & =-\frac{i \alpha}{w^{2}+\alpha^{2}} \sqrt{ \pm \zeta}
\end{aligned}
$$




$$
\begin{aligned}
\left|w^{2}+\alpha^{2}\right| & =\sqrt{\left(x^{2}+(\tau+\alpha)^{2}\right)\left(x^{2}+(\tau-\alpha)^{2}\right)} \\
& =\sqrt{\left(x^{2}+\tau^{2}-\alpha^{2}\right)^{2}+(2 x \alpha)^{2}} \\
\left|\frac{d \sqrt{ \pm \zeta}}{d w}\right|^{2} & =\frac{\alpha^{2}}{\left(x^{2}+(\tau-\alpha)^{2}\right) \sqrt{\left(x^{2}+\tau^{2}-\alpha^{2}\right)^{2}+(2 x \alpha)^{2}}}
\end{aligned}
$$

For simplicity we define $R, A_{ \pm}$as

$$
\begin{aligned}
R & =\sqrt{\left(x^{2}+\tau^{2}-\alpha^{2}\right)^{2}+(2 x \alpha)^{2}} \\
A_{ \pm} & =\sqrt{\left(x^{2}+\tau^{2}-\alpha^{2}\right)^{2}+(2 x \alpha)^{2}} \pm\left(x^{2}+\tau^{2}-\alpha^{2}\right) .
\end{aligned}
$$

And we get more results,

$$
\begin{aligned}
\sqrt{\zeta} & = \begin{cases}\frac{\sqrt{A_{+}}+i \sqrt{A_{-}}}{\sqrt{\left.2 x^{2}+(\tau-\alpha)^{2}\right)}} & (x>0) \\
\frac{\sqrt{A_{+}}-i \sqrt{A_{-}}}{\sqrt{2\left(x^{2}+(\tau-\alpha)^{2}\right)}} & (x<0) .\end{cases} \\
\sqrt{-\zeta} & = \begin{cases}-i \sqrt{\zeta} & (x>0) \\
i \sqrt{\zeta} \quad(x<0) .\end{cases} \\
\operatorname{Im}(i \sqrt{ \pm \zeta}) & =\sqrt{\frac{A_{ \pm}}{2\left(x^{2}+(\tau-\alpha)^{2}\right)}} \\
(\operatorname{Im}(i \sqrt{ \pm \zeta}))^{2} & =\frac{\sqrt{\left(x^{2}+\tau^{2}-\alpha^{2}\right)^{2}+(2 x \alpha)^{2}} \pm\left(x^{2}+\tau^{2}-\alpha^{2}\right)}{2\left(x^{2}+(\tau-\alpha)^{2}\right)}
\end{aligned}
$$

Also we can compute $\left|f\left(w_{a}\right)-f\left(w_{b}\right)\right|^{4}$ in the connected EE. If $a, b>0$

$$
\left|\sqrt{ \pm \zeta_{a}}-\sqrt{ \pm \zeta_{b}}\right|^{4}=\frac{\left|\left(\sqrt{A_{+a}}+i \sqrt{A_{-a}}\right) \sqrt{b^{2}+(\tau-\alpha)^{2}}-\left(\sqrt{A_{+b}}+i \sqrt{A_{-b}}\right) \sqrt{a^{2}+(\tau-\alpha)^{2}}\right|^{4}}{4\left(a^{2}+(\tau-\alpha)^{2}\right)^{2}\left(b^{2}+(\tau-\alpha)^{2}\right)^{2}}
$$

If $a<0<b$,

$$
\left|\sqrt{ \pm \zeta_{a}}-\sqrt{ \pm \zeta_{b}}\right|^{4}=\frac{\left|\left(\sqrt{A_{+a}}-i \sqrt{A_{-a}}\right) \sqrt{b^{2}+(\tau-\alpha)^{2}} \mp\left(\sqrt{A_{+b}}+i \sqrt{A_{-b}}\right) \sqrt{a^{2}+(\tau-\alpha)^{2}}\right|^{4}}{4\left(a^{2}+(\tau-\alpha)^{2}\right)^{2}\left(b^{2}+(\tau-\alpha)^{2}\right)^{2}}
$$

Here we write $\zeta(x, \tau)=\zeta_{x}, A_{ \pm}(x, \tau)=A_{ \pm x}$.

Now we can derive Euclidean HEE for the connected/disconnected geodesics, with $R(x, \tau)=R_{x}$,

$$
S_{A, \pm}^{\mathrm{con}}=\frac{c}{12} \log \begin{cases}\frac{R_{a} R_{b}\left(Q-\left(\sqrt{A_{+a} A_{+b}}+\sqrt{A_{-a} A_{-b}}\right)\right)^{2}}{\alpha^{4} \epsilon^{4}} & (a, b>0) \\ \frac{R_{a} R_{b}\left(Q \mp\left(\sqrt{A_{+a} A_{+b}}-\sqrt{A_{-a} A_{-b}}\right)\right)^{2}}{\alpha^{4} \epsilon^{4}} & (a<0<b)\end{cases}
$$




$$
\begin{aligned}
Q & =\sqrt{\left(b^{2}+(\tau-\alpha)^{2}\right)\left(a^{2}+(\tau+\alpha)^{2}\right)}+(a \leftrightarrow b) \\
& =\sqrt{2} \sqrt{\left(a^{2}+\tau^{2}+\alpha^{2}\right)\left(b^{2}+\tau^{2}+\alpha^{2}\right)-(2 \tau \alpha)^{2}+R_{a} R_{b}} \\
S_{A, \pm}^{\text {dis }} & =\frac{c}{12} \log \frac{4 R_{a} R_{b} A_{ \pm a} A_{ \pm b}}{\alpha^{4} \epsilon^{4}}
\end{aligned}
$$

We do analytic continuation to the real time $\tau \rightarrow i t$, and then we approximate $x, t, \mid x^{2}-$ $t^{2} \mid \gg \alpha$. Under this approximation we get

$$
\begin{aligned}
R_{x} & \sim\left|x^{2}-t^{2}\right|+\frac{x^{2}+t^{2}}{\left|x^{2}-t^{2}\right|} \alpha^{2} \\
A_{ \pm x} & \sim\left(\left|x^{2}-t^{2}\right| \pm\left(x^{2}-t^{2}\right)\right)+\left(\frac{x^{2}+t^{2}}{\left|x^{2}-t^{2}\right|} \mp 1\right) \alpha^{2}
\end{aligned}
$$

Consequently we derive the Lorentzian HEE formula. Here we write the results only in splitting quench case (i.e. $S_{A,+}$ case),

$$
S_{A,+}^{\text {dis }} \sim \frac{c}{6} \log \left\{\begin{array}{l}
\frac{4\left(a^{2}-t^{2}\right)\left(b^{2}-t^{2}\right)}{\alpha^{2} \epsilon^{2}} \quad(t<|a|<|b|) \\
\frac{4|a|\left(b^{2}-t^{2}\right)}{\alpha \epsilon^{2}} \quad(|a|<t<|b|) \\
\frac{4|a||b|}{\epsilon^{2}} \quad(|a|<|b|<t)
\end{array}\right.
$$

And for connected HEE we have to consider the sign of $a$. If $a, b>0$

$$
S_{A,+}^{\text {con }} \sim \frac{c}{6} \log \left\{\begin{array}{l}
\frac{(b-a)^{2}}{\epsilon^{2}} \quad(0<t<a, b<t) \\
\frac{2(b-a)(t-a)(b-t)}{\alpha \epsilon^{2}} \quad(a<t<b)
\end{array}\right.
$$

If $a<0<b,-a<b$,

$$
S_{A,+}^{\text {con }} \sim \frac{c}{6} \log \begin{cases}\frac{(b-a)^{2}}{\epsilon^{2}} \quad(0<t<-a) \\ \frac{2(b-a)(t+a)(b+t)}{\alpha \epsilon^{2}} \quad(-a<t<b) \\ \frac{4\left(t^{2}-a^{2}\right)\left(t^{2}-b^{2}\right)}{\alpha^{2} \epsilon^{2}} \quad(b<t)\end{cases}
$$

These give the results (4.13), (4.14) and (4.15). Similarly we can get the results for the holographic joining quenches (5.7), (5.8) and (5.9).

Open Access. This article is distributed under the terms of the Creative Commons Attribution License (CC-BY 4.0), which permits any use, distribution and reproduction in any medium, provided the original author(s) and source are credited. 


\section{References}

[1] J.M. Maldacena, The large $N$ limit of superconformal field theories and supergravity, Int. J. Theor. Phys. 38 (1999) 1113 [Adv. Theor. Math. Phys. 2 (1998) 231] [hep-th/9711200] [INSPIRE].

[2] S.S. Gubser, I.R. Klebanov and A.M. Polyakov, Gauge theory correlators from noncritical string theory, Phys. Lett. B 428 (1998) 105 [hep-th/9802109] [INSPIRE].

[3] E. Witten, Anti-de Sitter space and holography, Adv. Theor. Math. Phys. 2 (1998) 253 [hep-th/9802150] [InSPIRE].

[4] L. Bombelli, R.K. Koul, J. Lee and R.D. Sorkin, A quantum source of entropy for black holes, Phys. Rev. D 34 (1986) 373 [InSPIRE].

[5] M. Srednicki, Entropy and area, Phys. Rev. Lett. 71 (1993) 666 [hep-th/9303048] [INSPIRE].

[6] C. Holzhey, F. Larsen and F. Wilczek, Geometric and renormalized entropy in conformal field theory, Nucl. Phys. B 424 (1994) 443 [hep-th/9403108] [INSPIRE].

[7] P. Calabrese and J.L. Cardy, Entanglement entropy and quantum field theory, J. Stat. Mech. 0406 (2004) P06002 [hep-th/0405152] [INSPIRE].

[8] P. Calabrese and J. Cardy, Entanglement entropy and conformal field theory, J. Phys. A 42 (2009) 504005 [arXiv:0905.4013] [INSPIRE].

[9] H. Casini, C.D. Fosco and M. Huerta, Entanglement and alpha entropies for a massive Dirac field in two dimensions, J. Stat. Mech. 0507 (2005) P07007 [cond-mat/0505563] [INSPIRE].

[10] H. Casini and M. Huerta, Entanglement entropy in free quantum field theory, J. Phys. A 42 (2009) 504007 [arXiv:0905.2562] [INSPIRE].

[11] M. Nozaki, T. Numasawa and T. Takayanagi, Quantum entanglement of local operators in conformal field theories, Phys. Rev. Lett. 112 (2014) 111602 [arXiv:1401.0539] [INSPIRE].

[12] M. Nozaki, Notes on quantum entanglement of local operators, JHEP 10 (2014) 147 [arXiv:1405.5875] [INSPIRE].

[13] P. Calabrese and J. Cardy, Entanglement and correlation functions following a local quench: a conformal field theory approach, J. Stat. Mech. 0710 (2007) P10004 [arXiv:0708.3750] [INSPIRE].

[14] M. Nozaki, T. Numasawa and T. Takayanagi, Holographic local quenches and entanglement density, JHEP 05 (2013) 080 [arXiv: 1302.5703] [INSPIRE].

[15] T. Takayanagi, Holographic dual of BCFT, Phys. Rev. Lett. 107 (2011) 101602 [arXiv:1105.5165] [INSPIRE].

[16] M. Fujita, T. Takayanagi and E. Tonni, Aspects of AdS/BCFT, JHEP 11 (2011) 043 [arXiv:1108.5152] [INSPIRE].

[17] T. Ugajin, Two dimensional quantum quenches and holography, arXiv:1311.2562 [INSPIRE].

[18] T. Numasawa, N. Shiba, T. Takayanagi and K. Watanabe, EPR pairs, local projections and quantum teleportation in holography, JHEP 08 (2016) 077 [arXiv: 1604.01772] [INSPIRE].

[19] B. Swingle, Entanglement renormalization and holography, Phys. Rev. D 86 (2012) 065007 [arXiv:0905.1317] [INSPIRE].

[20] G. Vidal, Class of quantum many-body states that can be efficiently simulated, Phys. Rev. Lett. 101 (2008) 110501 [INSPIRE]. 
[21] G. Vidal, Entanglement renormalization, Phys. Rev. Lett. 99 (2007) 220405 [cond-mat/0512165] [INSPIRE].

[22] F. Pastawski, B. Yoshida, D. Harlow and J. Preskill, Holographic quantum error-correcting codes: Toy models for the bulk/boundary correspondence, JHEP 06 (2015) 149 [arXiv: 1503.06237] [INSPIRE].

[23] P. Hayden et al., Holographic duality from random tensor networks, JHEP 11 (2016) 009 [arXiv: 1601.01694] [INSPIRE].

[24] J. Haegeman, T.J. Osborne, H. Verschelde and F. Verstraete, Entanglement renormalization for quantum fields in real space, Phys. Rev. Lett. 110 (2013) 100402 [arXiv:1102.5524] [INSPIRE].

[25] M. Nozaki, S. Ryu and T. Takayanagi, Holographic geometry of entanglement renormalization in quantum field theories, JHEP 10 (2012) 193 [arXiv: 1208.3469] [INSPIRE].

[26] A. Mollabashi, M. Nozaki, S. Ryu and T. Takayanagi, Holographic geometry of cMERA for quantum quenches and finite temperature, JHEP 03 (2014) 098 [arXiv:1311.6095] [INSPIRE].

[27] G. Evenbly and G. Vidal, Tensor network renormalization, Phys. Rev. Lett. 115 (2015) 180405 [arXiv: 1412.0732].

[28] G. Evenbly and G. Vidal, Tensor network renormalization yields the multi-scale entanglement renormalization ansatz, Phys. Rev. Lett. 115 (2015) 200401 [arXiv:1502.05385].

[29] M. Miyaji, T. Takayanagi and K. Watanabe, From path integrals to tensor networks for the AdS/CFT correspondence, Phys. Rev. D 95 (2017) 066004 [arXiv:1609.04645] [INSPIRE].

[30] P. Caputa et al., Anti-de Sitter space from optimization of path integrals in conformal field theories, Phys. Rev. Lett. 119 (2017) 071602 [arXiv:1703.00456] [INSPIRE].

[31] P. Caputa et al., Liouville action as path-integral complexity: from continuous tensor networks to AdS/CFT, JHEP 11 (2017) 097 [arXiv: 1706.07056] [INSPIRE].

[32] A. Milsted and G. Vidal, Tensor networks as path integral geometry, arXiv:1807.02501 [INSPIRE].

[33] A. Milsted and G. Vidal, Tensor networks as conformal transformations, arXiv:1805.12524 [INSPIRE].

[34] T. Takayanagi, Holographic spacetimes as quantum circuits of path-integrations, JHEP 12 (2018) 048 [arXiv: 1808.09072] [INSPIRE].

[35] M.M. Roberts, Time evolution of entanglement entropy from a pulse, JHEP 12 (2012) 027 [arXiv: 1204.1982] [INSPIRE].

[36] S. Ryu and T. Takayanagi, Holographic derivation of entanglement entropy from AdS/CFT, Phys. Rev. Lett. 96 (2006) 181602 [hep-th/0603001] [INSPIRE].

[37] S. Ryu and T. Takayanagi, Aspects of holographic entanglement entropy, JHEP 08 (2006) 045 [hep-th/0605073] [inSPIRE].

[38] V.E. Hubeny, M. Rangamani and T. Takayanagi, A covariant holographic entanglement entropy proposal, JHEP 07 (2007) 062 [arXiv:0705.0016] [INSPIRE].

[39] A. Karch and L. Randall, Open and closed string interpretation of SUSY CFT's on branes with boundaries, JHEP 06 (2001) 063 [hep-th/0105132] [INSPIRE]. 
[40] M. Nozaki, T. Takayanagi and T. Ugajin, Central charges for BCFTs and holography, JHEP 06 (2012) 066 [arXiv: 1205.1573] [INSPIRE].

[41] R.-X. Miao and C.-S. Chu, Universality for shape dependence of Casimir effects from Weyl anomaly, JHEP 03 (2018) 046 [arXiv:1706.09652] [INSPIRE].

[42] I. Affleck and A.W.W. Ludwig, Universal noninteger 'ground state degeneracy' in critical quantum systems, Phys. Rev. Lett. 67 (1991) 161 [INSPIRE].

[43] D. Seminara, J. Sisti and E. Tonni, Corner contributions to holographic entanglement entropy in $A d S_{4} / B C F T_{3}, J H E P 11$ (2017) 076 [arXiv: 1708.05080] [INSPIRE].

[44] D. Seminara, J. Sisti and E. Tonni, Holographic entanglement entropy in $A d S_{4} / B C F T_{3}$ and the Willmore functional, JHEP 08 (2018) 164 [arXiv: 1805.11551] [INSPIRE].

[45] N. Kobayashi, T. Nishioka, Y. Sato and K. Watanabe, Towards a C-theorem in defect CFT, JHEP 01 (2019) 039 [arXiv:1810.06995] [INSPIRE].

[46] C.T. Asplund and A. Bernamonti, Mutual information after a local quench in conformal field theory, Phys. Rev. D 89 (2014) 066015 [arXiv:1311.4173] [InSPIRE].

[47] J. Bhattacharya, M. Nozaki, T. Takayanagi and T. Ugajin, Thermodynamical property of entanglement entropy for excited states, Phys. Rev. Lett. 110 (2013) 091602 [arXiv: 1212.1164] [INSPIRE].

[48] D.D. Blanco, H. Casini, L.-Y. Hung and R.C. Myers, Relative entropy and holography, JHEP 08 (2013) 060 [arXiv: 1305.3182] [inSPIRE].

[49] G. Wong, I. Klich, L.A. Pando Zayas and D. Vaman, Entanglement temperature and entanglement entropy of excited states, JHEP 12 (2013) 020 [arXiv: 1305.3291] [INSPIRE].

[50] P. Calabrese and J.L. Cardy, Evolution of entanglement entropy in one-dimensional systems, J. Stat. Mech. 0504 (2005) P04010 [cond-mat/0503393] [INSPIRE].

[51] J.L. Cardy, Boundary conditions, fusion rules and the Verlinde formula, Nucl. Phys. B 324 (1989) 581 [INSPIRE].

[52] N. Ishibashi, The boundary and crosscap states in conformal field theories, Mod. Phys. Lett. A 4 (1989) 251 [INSPIRE].

[53] T. Takayanagi and T. Ugajin, Measuring black hole formations by entanglement entropy via coarse-graining, JHEP 11 (2010) 054 [arXiv: 1008.3439] [INSPIRE].

[54] J. Abajo-Arrastia, J. Aparicio and E. Lopez, Holographic evolution of entanglement entropy, JHEP 11 (2010) 149 [arXiv: 1006.4090] [INSPIRE].

[55] V. Balasubramanian et al., Thermalization of strongly coupled field theories, Phys. Rev. Lett. 106 (2011) 191601 [arXiv: 1012.4753] [INSPIRE].

[56] T. Hartman and J. Maldacena, Time evolution of entanglement entropy from black hole interiors, JHEP 05 (2013) 014 [arXiv: 1303.1080] [INSPIRE].

[57] C.T. Asplund, A. Bernamonti, F. Galli and T. Hartman, Entanglement scrambling in $2 d$ conformal field theory, JHEP 09 (2015) 110 [arXiv:1506.03772] [INSPIRE].

[58] S. He, T. Numasawa, T. Takayanagi and K. Watanabe, Quantum dimension as entanglement entropy in two dimensional conformal field theories, Phys. Rev. D 90 (2014) 041701 [arXiv: 1403.0702] [INSPIRE]. 
[59] P. Caputa, J. Simón, A. Štikonas and T. Takayanagi, Quantum entanglement of localized excited states at finite temperature, JHEP 01 (2015) 102 [arXiv:1410.2287] [INSPIRE].

[60] J. de Boer et al., Higher spin entanglement and $\mathcal{W}_{N}$ conformal blocks, JHEP 07 (2015) 168 [arXiv: 1412.7520] [INSPIRE].

[61] W.-Z. Guo and S. He, Rényi entropy of locally excited states with thermal and boundary effect in 2D CFTs, JHEP 04 (2015) 099 [arXiv:1501.00757] [INSPIRE].

[62] B. Chen, W.-Z. Guo, S. He and J.-q. Wu, Entanglement entropy for descendent local operators in 2D CFTs, JHEP 10 (2015) 173 [arXiv: 1507.01157] [INSPIRE].

[63] M. Nozaki, T. Numasawa and S. Matsuura, Quantum entanglement of fermionic local operators, JHEP 02 (2016) 150 [arXiv: 1507.04352] [INSPIRE].

[64] P. Caputa and A. Veliz-Osorio, Entanglement constant for conformal families, Phys. Rev. D 92 (2015) 065010 [arXiv: 1507.00582] [INSPIRE].

[65] P. Caputa et al., Scrambling time from local perturbations of the eternal BTZ black hole, JHEP 08 (2015) 011 [arXiv: 1503.08161] [INSPIRE].

[66] M. Rangamani, M. Rozali and A. Vincart-Emard, Dynamics of holographic entanglement entropy following a local quench, JHEP 04 (2016) 069 [arXiv:1512.03478] [INSPIRE].

[67] A. Sivaramakrishnan, Localized excitations from localized unitary operators, Annals Phys. 381 (2017) 41 [arXiv:1604.00965] [INSPIRE].

[68] P. Caputa and M.M. Rams, Quantum dimensions from local operator excitations in the Ising model, J. Phys. A 50 (2017) 055002 [arXiv: 1609. 02428] [INSPIRE].

[69] T. Numasawa, Scattering effect on entanglement propagation in RCFTs, JHEP 12 (2016) 061 [arXiv: 1610.06181] [INSPIRE].

[70] M. Nozaki and N. Watamura, Quantum entanglement of locally excited states in Maxwell theory, JHEP 12 (2016) 069 [arXiv: 1606.07076] [InSPIRE].

[71] J.R. David, S. Khetrapal and S.P. Kumar, Universal corrections to entanglement entropy of local quantum quenches, JHEP 08 (2016) 127 [arXiv: 1605. 05987] [INSPIRE].

[72] P. Caputa, Y. Kusuki, T. Takayanagi and K. Watanabe, Evolution of entanglement entropy in orbifold CFTs, J. Phys. A 50 (2017) 244001 [arXiv:1701.03110] [INSPIRE].

[73] M. Nozaki and N. Watamura, Correspondence between entanglement growth and probability distribution of quasiparticles, Phys. Rev. D 96 (2017) 025019 [arXiv:1703.06589] [INSPIRE].

[74] A. Jahn and T. Takayanagi, Holographic entanglement entropy of local quenches in $A d S_{4} / C_{F T}$ : a finite-element approach, J. Phys. A 51 (2018) 015401 [arXiv:1705.04705] [INSPIRE].

[75] S. He, Conformal bootstrap to Rényi entropy in $2 D$ Liouville and super-Liouville CFTs, Phys. Rev. D 99 (2019) 026005 [arXiv:1711.00624] [INSPIRE].

[76] Y. Kusuki and T. Takayanagi, Renyi entropy for local quenches in $2 D$ CFT from numerical conformal blocks, JHEP 01 (2018) 115 [arXiv:1711.09913] [INSPIRE].

[77] Y. Kusuki, Light cone bootstrap in general $2 D$ CFTs and entanglement from light cone singularity, JHEP 01 (2019) 025 [arXiv:1810.01335] [INSPIRE].

[78] P. Caputa, M. Nozaki and T. Takayanagi, Entanglement of local operators in large- $N$ conformal field theories, PTEP 2014 (2014) 093B06 [arXiv: 1405. 5946] [INSPIRE]. 
[79] C.T. Asplund, A. Bernamonti, F. Galli and T. Hartman, Holographic entanglement entropy from 2D CFT: heavy states and local quenches, JHEP 02 (2015) 171 [arXiv:1410.1392] [INSPIRE].

[80] T. Azeyanagi, T. Nishioka and T. Takayanagi, Near extremal black hole entropy as entanglement entropy via $A d S_{2} / C F T_{1}$, Phys. Rev. D 77 (2008) 064005 [arXiv:0710.2956] [INSPIRE].

[81] A.F. Astaneh and A.E. Mosaffa, Quantum local quench, AdS/BCFT and yo-yo string, JHEP 05 (2015) 107 [arXiv: 1405.5469] [INSPIRE].

[82] G.T. Horowitz and N. Itzhaki, Black holes, shock waves and causality in the AdS/CFT correspondence, JHEP 02 (1999) 010 [hep-th/9901012] [INSPIRE].

[83] G. Evenbly and G. Vidal, Algorithms for entanglement renormalization: boundaries, impurities and interfaces, J. Stat. Phys. 157 (2014) 931 [arXiv:1312.0303].

[84] M. Van Raamsdonk, Building up spacetime with quantum entanglement II: it from BC-bit, arXiv: 1809.01197 [INSPIRE].

[85] M. Miyaji, S. Ryu, T. Takayanagi and X. Wen, Boundary states as holographic duals of trivial spacetimes, JHEP 05 (2015) 152 [arXiv:1412.6226] [INSPIRE].

[86] T. Numasawa, Holographic complexity for disentangled states, arXiv:1811.03597 [INSPIRE].

[87] D.D. Blanco and H. Casini, Entanglement entropy for non-coplanar regions in quantum field theory, Class. Quant. Grav. 28 (2011) 215015 [arXiv:1103.4400] [INSPIRE]. 